\title{
Conservative versus accelerated rehabilitation after rotator cuff repair: a systematic review and meta-analysis
}

Umile Giuseppe Longo ${ }^{{ }^{*}} \mathbb{D}$, Laura Risi Ambrogioni ${ }^{1}$, Alessandra Berton ${ }^{1}$, Vincenzo Candela ${ }^{1}$, Filippo Migliorini ${ }^{1}$, Arianna Carnevale ${ }^{2}$, Emiliano Schena ${ }^{2}$, Ara Nazariann ${ }^{3}$, Joseph DeAngelis ${ }^{3}$ and Vincenzo Denaro ${ }^{1}$

\begin{abstract}
Background: The purpose of this systematic review and meta-analysis is to compare the conservative and accelerated rehabilitation protocols in patients who underwent arthroscopic rotator cuff repair in terms of clinical outcomes and range of motions at 3,6, 12, and 24-month follow-up.

Methods: According to PRISMA guidelines, a systematic review of the literature was performed. For each included article, the following data has been extracted: authors, year, study design, level of evidence, demographic characteristics, follow-up, clinical outcomes, range of motions, and retear events. A meta-analysis was performed to compare accelerated versus conservative rehabilitation protocols after arthroscopic rotator cuff repair. The retear rate, postoperative Constant-Murley score and range of motions at 3, 6, 12, and 24 months of follow-up were the outcomes measured.

Results: The search strategy yielded 16 level I-II clinical studies. A total of 1424 patients, with 732 patients and 692 in the accelerated and conservative group, were included. The average age (mean \pm standard deviation) was $56.1 \pm$ 8.7 and $56.6 \pm 9$ in the accelerated and conservative group. The mean follow-up was 12.5 months, ranging from 2 to 24 months. The meta-analysis showed no statistically significant differences in terms of retear rate between the groups $(P=0.29)$. The superiority of the accelerated group was demonstrated in terms of external rotation $(P<0.05)$ at 3-month follow-up; in terms of forward elevation, external rotation, abduction $(P<0.05)$, but not in terms of Constant-Murley score at 6-month follow-up; in terms of forward elevation $(P<0.05)$ at 12 -month follow-up. No significant differences between the two group were highlighted at 24-month follow-up.

Conclusions: No statistically significant differences in the retear rate among the accelerated and conservative group have been demonstrated. On the other hand, statistically and clinically significant differences were found in terms of external rotation at 3 and 6 months of follow-up in favour of the accelerated group. However, no differences between the two groups were detected at 24 months follow-up.
\end{abstract}

Keywords: Conservative, Accelerated, Rehabilitation, Protocol, Rotator cuff, Rotator cuff repair

\footnotetext{
* Correspondence: g.longo@unicampus.it

'Department of Orthopaedic and Trauma Surgery, Campus Bio-Medico

University, Via Alvaro del Portillo, 200, Rome, Trigoria 00128, Italy

Full list of author information is available at the end of the article
}

C C The Author(s). 2021 Open Access This article is licensed under a Creative Commons Attribution 4.0 International License, which permits use, sharing, adaptation, distribution and reproduction in any medium or format, as long as you give appropriate credit to the original author(s) and the source, provide a link to the Creative Commons licence, and indicate if changes were made. The images or other third party material in this article are included in the article's Creative Commons licence, unless indicated otherwise in a credit line to the material. If material is not included in the article's Creative Commons licence and your intended use is not permitted by statutory regulation or exceeds the permitted use, you will need to obtain permission directly from the copyright holder. To view a copy of this licence, visit http://creativecommons.org/licenses/by/4.0/ The Creative Commons Public Domain Dedication waiver (http://creativecommons.org/publicdomain/zero/1.0/) applies to the data made available in this article, unless otherwise stated in a credit line to the data. 


\section{Background}

Rotator cuff (RC) tears represent a significant reason for orthopaedic examination due to their negative impact on the working class's quality of life [1-4]. The broad interest in $\mathrm{RC}$ disease is because pathogenesis is still unclear [5-14], the management remains challenging [1518 ], and the patient lifestyle can be scanty [19-23].

Management of RC tears depends on the tear's characteristics, such as tear severity, location and extent of the injury, tendon retraction, and muscle condition. Treatment of RC can be conservative or surgical [24-28]. Surgery is performed after trauma or a conservative treatment failure to improve shoulder function and relieve pain [29-31]. Among surgical techniques, arthroscopy is the most commonly performed because of minor trauma to the deltoid due to smaller incisions, less postoperative pain, and the possibility of addressing concomitant disorders [32-45]. As a result of the increasing incidence of $\mathrm{RC}$ ruptures and the risk of failure after surgical repair, questions have been raised regarding the cost-benefit ratio of surgical treatment compared with conservative management $[2,3,46-53]$. The immobilisation with the sling, the physiotherapist's role, and the best postoperative week for reintroducing shoulder movement have been widely investigated $[24,29$, 54-73]. The best rehabilitation protocol aims to restore shoulder function, allowing the tendon healing process to prevent retear events [60, 74]. To achieve this goal, both conservative and accelerated rehabilitation protocols have been proposed. The first protocol requires a restrained arm during the first six weeks. The rationale for conservative rehabilitation protocol is based on evidence from animal studies showing that the tendon healing process takes 4 to 16 weeks $[75,76]$. It is common practice to apply a sling immobilisation in abduction wrap to reduce potential stress at the suture level and to improve the blood flow quantity at the scar [42, 77-83]. However, there are concerns about stiffness after prolonged immobilisation [84-86]. Recent studies have demonstrated that prolonged immobilisation may result in biochemical tissue changes, such as variation in water and glycosaminoglycans concentration, the regularity of collagen cross-bridges, fatty infiltration, and fibres

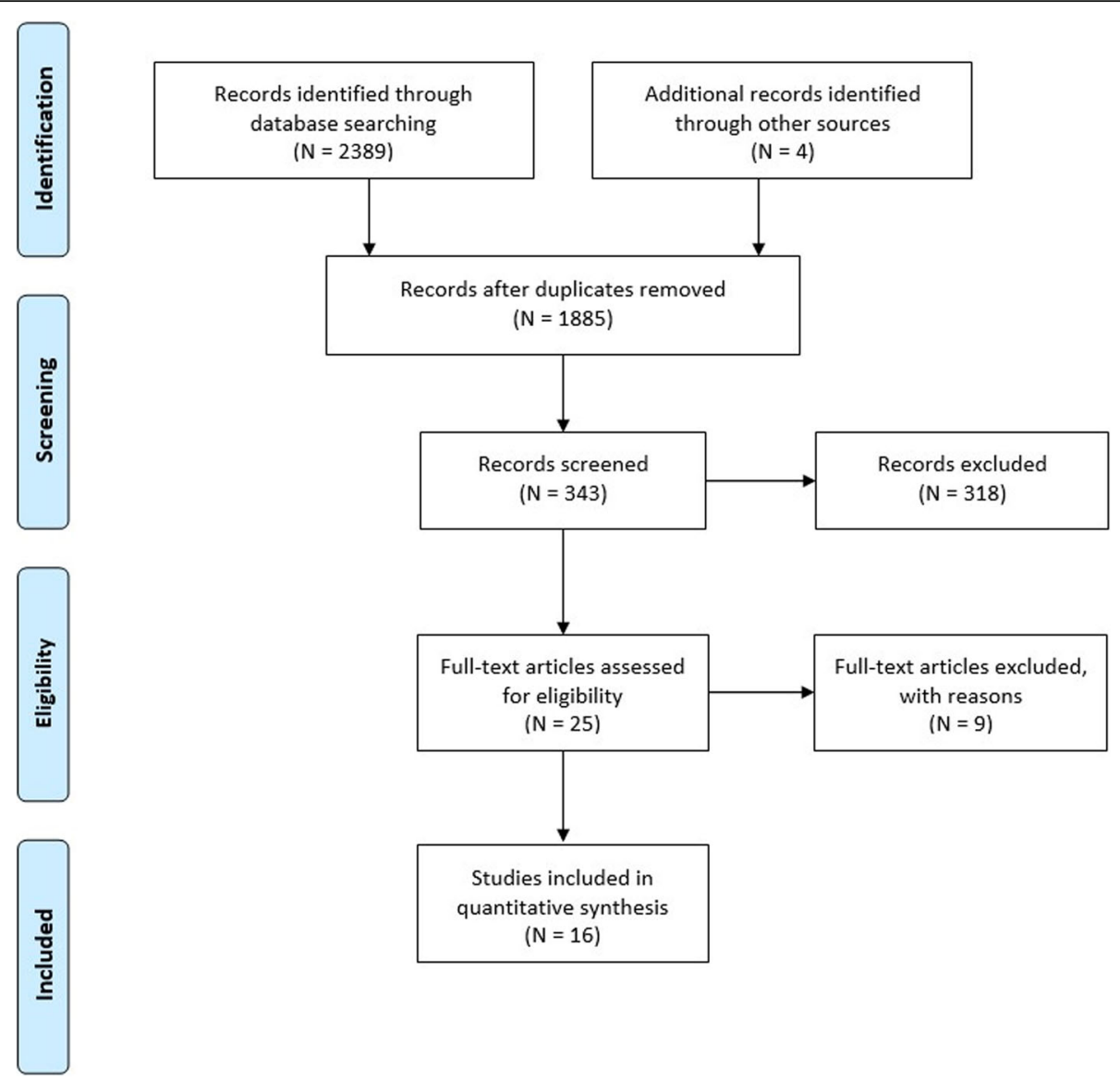

Fig. 1 Preferred Reporting Items for Systematic Reviews and Meta-Analyses (PRISMA) diagram representing individual study inclusion after applying the study algorithm 
Table 1 Demographics

\begin{tabular}{|c|c|c|c|c|c|}
\hline $\begin{array}{l}\text { Authors } \\
\text { (Year, level of } \\
\text { evidence) }\end{array}$ & $\begin{array}{l}\text { No of final patients (No of initial patients - } \\
\text { losses at follow-up) }{ }^{\text {a }}\end{array}$ & $\begin{array}{l}\text { No of patient in the accelerated group (AG) and } \\
\text { conservative group (CG) }\end{array}$ & Sex & $\begin{array}{l}\text { Average age (mean } \pm \\
\text { standard deviation) }\end{array}$ & $\begin{array}{l}\text { Follow-up } \\
\text { (months) }\end{array}$ \\
\hline $\begin{array}{l}\text { Arndt et al. [61] } \\
(2012, \text { II) }\end{array}$ & $92(100-8)$ & $\begin{array}{l}\text { AG (49) } \\
\text { CG (43) }\end{array}$ & M 34; F 58 & $54.6 \pm 9.8$ & 16 \\
\hline $\begin{array}{l}\text { Cuff et al. [70] } \\
(2012,1)\end{array}$ & $68(68-0)$ & $\begin{array}{l}\text { AG (33) } \\
\text { CG (35) }\end{array}$ & $\begin{array}{l}\text { AG (M 18; } \\
\text { F 15) } \\
\text { CG (M 20; } \\
\text { F 15) }\end{array}$ & $\begin{array}{l}\text { AG: } 54.8 \pm 15.9 \\
\text { CG: } 56.3 \pm 15.6\end{array}$ & 12 \\
\hline $\begin{array}{l}\text { De Roo et al. } \\
{[110]} \\
(2015,1)\end{array}$ & $130(130-0)$ & $\begin{array}{l}\text { AG (79) } \\
\text { CG (51) }\end{array}$ & $\begin{array}{l}\text { AG (M 48; } \\
\text { F 31) } \\
\text { CG (M 41; } \\
\text { F 10) }\end{array}$ & $\begin{array}{l}\text { AG: } 64.6 \pm 10.0 \\
\text { CG: } 65.1 \pm 9.7\end{array}$ & 4 \\
\hline $\begin{array}{l}\text { Düzgün et al. } \\
\text { [71] } \\
(2011, \|)\end{array}$ & $29(29-0)$ & $\begin{array}{l}\text { AG (13) } \\
\text { CG (16) }\end{array}$ & M 3; F 26 & $\begin{array}{l}\text { AG: } 55.9 \pm 7.8 \\
\text { CG: } 56.6 \pm 11\end{array}$ & 4 \\
\hline $\begin{array}{l}\text { Düzgün et al. } \\
{[29]} \\
(2014, \text { II) }\end{array}$ & $40(42-2)$ & $\begin{array}{l}\text { AG (19) } \\
\text { CG (21) }\end{array}$ & $\begin{array}{l}\text { AG (M 2; } \\
\text { F 17) } \\
\text { CG (M 4; F } \\
17)\end{array}$ & $\begin{array}{l}\text { AG: } 57.7 \pm 7.8 \\
\text { CG: } 57.2 \pm 10.1\end{array}$ & 6 \\
\hline $\begin{array}{l}\text { Jenssen et al. } \\
{[73]} \\
(2018,1)\end{array}$ & $118(120-2)$ & $\begin{array}{l}\text { AG (60) } \\
\text { CG (58) }\end{array}$ & $\begin{array}{l}\text { AG (M 37; } \\
\text { F 23) } \\
\text { CG (M 32; } \\
\text { F 26) }\end{array}$ & $\begin{array}{l}\text { AG: } 53.8 \pm 10.1 \\
\text { CG: } 54.3 \pm 11.3\end{array}$ & 12 \\
\hline $\begin{array}{l}\text { Keener et al. [63] } \\
(2014,1)\end{array}$ & $114(129-15)$ & $\begin{array}{l}\text { AG (61) } \\
\text { CG (53) }\end{array}$ & - & $\begin{array}{l}\text { AG: } 54.8 \pm 6.3 \\
\text { CG: } 55.8 \pm 6.3\end{array}$ & 24 \\
\hline $\begin{array}{l}\text { Kim et al. [64] } \\
(2012,1)\end{array}$ & $105(117-12)$ & $\begin{array}{l}\text { AG (56) } \\
\text { CG (49) }\end{array}$ & $\begin{array}{l}\text { AG (M 26; } \\
\text { F } 30) \\
\text { CG (M 18; } \\
\text { F 31) }\end{array}$ & $\begin{array}{l}\text { AG: } 60.1 \pm 9 \\
\text { CG: } 60 \pm 10.4\end{array}$ & 12 \\
\hline $\begin{array}{l}\text { Klintberg et al. } \\
{[72]} \\
(2009,11)\end{array}$ & $14(22-8)$ & $\begin{array}{l}\text { AG (7) } \\
\text { CG (7) }\end{array}$ & M 9; F 5 & $53.5 \pm 6.9$ & 24 \\
\hline $\begin{array}{l}\text { Koh. et al. [77] } \\
(2014,1)\end{array}$ & $88(100-12)$ & $\begin{array}{l}\text { AG (40) } \\
\text { CG (48) }\end{array}$ & M 44; F 44 & 59.9 & 2 \\
\hline $\begin{array}{l}\text { Lee et al. [67] } \\
(2012,11)\end{array}$ & $64(85-21)$ & $\begin{array}{l}\text { AG (30) } \\
\text { CG (34) }\end{array}$ & $\begin{array}{l}\text { AG (M 21; } \\
\text { F 9) } \\
\text { CG (M 20; } \\
\text { F 14) }\end{array}$ & $\begin{array}{l}\text { AG: } 53.5 \pm 7.8 \\
\text { CG: } 53.85 \pm 7.2\end{array}$ & 12 \\
\hline $\begin{array}{l}\text { Mazzocca et al. } \\
{[68]} \\
(2017, \text { II) }\end{array}$ & $58(73-15)$ & $\begin{array}{l}\text { AG (31) } \\
\text { CG (27) }\end{array}$ & $\begin{array}{l}\text { AG (M 21; } \\
\text { F 10) } \\
\text { CG (M 19; } \\
\text { F 8) }\end{array}$ & $\begin{array}{l}\text { AG: } 55 \pm 8 \\
\text { CG: } 54 \pm 7\end{array}$ & 12 \\
\hline $\begin{array}{l}\text { Raschhofer et al. } \\
{[69]} \\
(2017, \text { II) }\end{array}$ & $29(30-1)$ & $\begin{array}{l}\text { AG (14) } \\
\text { CG (15) }\end{array}$ & $\begin{array}{l}\text { AG (M 10; } \\
\text { F 4) } \\
\text { CG (M 9; F } \\
6 \text { ) }\end{array}$ & $\begin{array}{l}\text { AG: } 54.4 \pm 3.0 \\
\text { CG: } 58.4 \pm 3.5\end{array}$ & 6 \\
\hline $\begin{array}{l}\text { Sheps et al. [78] } \\
(2015, \text { II) }\end{array}$ & $165(189-24)$ & $\begin{array}{l}\text { AG (97) } \\
\text { CG (92) }\end{array}$ & $\begin{array}{l}\text { AG (M 57; } \\
\text { F 40) } \\
\text { CG (M 58; } \\
\text { F 34) }\end{array}$ & $\begin{array}{l}\text { AG: } 55.4 \\
\text { CG: } 54.9\end{array}$ & 24 \\
\hline $\begin{array}{l}\text { Sheps et al. [79] } \\
(2019,1)\end{array}$ & $206(206-0)$ & $\begin{array}{l}\text { AG (103) } \\
\text { CG (103) }\end{array}$ & $\begin{array}{l}\text { AG (M 65; } \\
\text { F 38) } \\
\text { CG (M 66; } \\
\text { F 37) }\end{array}$ & $\begin{array}{l}\text { AG: } 55.5 \\
\text { CG: } 56.2\end{array}$ & 24 \\
\hline $\begin{array}{l}\text { Tirefort et al. [80] } \\
(2019,1)\end{array}$ & $80(80-0)$ & $\begin{array}{l}\text { AG (40) } \\
\text { CG (40) }\end{array}$ & $\begin{array}{l}\text { AG (M 23; } \\
\text { F 17) } \\
\text { CG (M 14; } \\
\text { F 26) }\end{array}$ & $\begin{array}{l}\text { AG: } 53.5 \pm 11.0 \\
\text { CG: } 54.7 \pm 8.7\end{array}$ & 6 \\
\hline
\end{tabular}

orientation $[65,71]$. Therefore, in stiffness-prone patients, an accelerated protocol that gradually reintegrates movement before the sixth week is advocated to achieve better clinical results [87]. However, accelerated rehabilitation protocol seems to increase the risk of tendon retear that occur from 20 to $90 \%$ of cases [64, 80, 88-91].
Even though many systematic reviews are available, the evidence is limited for the low statistical sample and the high heterogeneity among the included studies [4, 53, 92-98]. Since further clinical trials with a longer follow-up have been published recently, this review aims to compare the rehabilitation protocol and the accelerated protocol at different time-points. Therefore, the 
Table 2 Clinical outcomes and range of motions at a maximum of follow-up

\begin{tabular}{|c|c|c|c|c|c|c|c|c|c|c|}
\hline \multirow{2}{*}{$\begin{array}{l}\text { Authors } \\
\text { (Year, level } \\
\text { of } \\
\text { evidence) }\end{array}$} & \multirow{2}{*}{$\begin{array}{l}\text { No of } \\
\text { patients in } \\
\text { the } \\
\text { accelerated } \\
\text { group (AG) } \\
\text { and } \\
\text { conservative } \\
\text { group (CG)a }\end{array}$} & \multicolumn{3}{|c|}{$\begin{array}{l}\text { Clinical outcomes } \\
\text { (Mean } \pm \text { standard deviation) }\end{array}$} & \multicolumn{6}{|c|}{$\begin{array}{l}\text { Range of motion } \\
\text { (Degrees } \pm \text { standard deviation) }\end{array}$} \\
\hline & & $\begin{array}{l}\text { Constant- } \\
\text { Murley } \\
\text { score }\end{array}$ & $\begin{array}{l}\text { Simple } \\
\text { shoulder } \\
\text { test }\end{array}$ & $\begin{array}{l}\text { ASES } \\
\text { score }\end{array}$ & $\begin{array}{l}\text { Forward } \\
\text { elevation }\end{array}$ & $\begin{array}{l}\text { Internal } \\
\text { rotation } \\
\text { at side }\end{array}$ & $\begin{array}{l}\text { External } \\
\text { rotation } \\
\text { at side }\end{array}$ & $\begin{array}{l}\text { Internal } \\
\text { rotation at } 90^{\circ} \\
\text { of abduction }\end{array}$ & $\begin{array}{l}\text { External } \\
\text { rotation at } 90^{\circ} \\
\text { of abduction }\end{array}$ & Abduction \\
\hline \multirow[t]{2}{*}{$\begin{array}{l}\text { Arndt et al. } \\
\text { [61] }(2012, \text { II) }\end{array}$} & AG (49) & $77.6 \pm 12.4$ & - & - & $\begin{array}{l}172.4 \pm \\
13.0\end{array}$ & - & $58.7 \pm 12.9$ & - & - & - \\
\hline & CG (43) & $69.7 \pm 18$ & - & - & $\begin{array}{l}163.3 \pm \\
25.1\end{array}$ & - & $49.1 \pm 18.0$ & - & - & - \\
\hline \multirow{2}{*}{$\begin{array}{l}\text { Cuff et al. } \\
\text { [70] }(2012,1)\end{array}$} & AG (33) & - & 11.1 & 91.1 & 174 & 94 & 46 & - & - & - \\
\hline & CG (35) & - & 11.1 & 92.8 & 173 & 91 & 45 & - & - & - \\
\hline \multirow{2}{*}{$\begin{array}{l}\text { De Roo } \\
\text { et al. }[110] \\
(2015, \mathrm{l})\end{array}$} & AG (79) & $85.9 \pm 15.7$ & $9.4 \pm 2.2$ & - & $\begin{array}{l}139.2 \pm \\
16.8\end{array}$ & - & $46.2 \pm 17.3$ & $62.5 \pm 16.8$ & $64.2 \pm 17.4$ & $128 \pm 20.2$ \\
\hline & CG (51) & $90.4 \pm 18.4$ & $9 \pm 2.5$ & - & $\begin{array}{l}141.3 \pm \\
17.7\end{array}$ & - & $46.3 \pm 14.7$ & $64.5 \pm 21.3$ & $63.53 \pm 16.60$ & $\begin{array}{l}129.6 \pm \\
18.7\end{array}$ \\
\hline \multirow{2}{*}{$\begin{array}{l}\text { Düzgün } \\
\text { et al. [71] } \\
\text { (2011, II) }\end{array}$} & AG (13) & - & - & - & - & - & - & - & - & - \\
\hline & CG (16) & - & - & - & - & - & - & - & - & - \\
\hline \multirow{2}{*}{$\begin{array}{l}\text { Düzgün } \\
\text { et al. [29] } \\
(2014, \text { II) }\end{array}$} & AG (19) & - & - & - & $\begin{array}{l}158.9 \pm \\
1.8\end{array}$ & $88.2 \pm 2.1$ & $86.3 \pm 2.4$ & - & - & $173.6 \pm 3.4$ \\
\hline & CG (21) & - & - & - & $153.7 \pm 4$ & $86.5 \pm 1.9$ & $84.1 \pm 2.2$ & - & - & $171 \pm 3.1$ \\
\hline \multirow{2}{*}{$\begin{array}{l}\text { Jenssen } \\
\text { et al. [73] } \\
(2018,1)\end{array}$} & AG (60) & $86 \pm 27$ & - & - & $151 \pm 15$ & $61 \pm 9$ & $64 \pm 12$ & - & - & $146 \pm 22$ \\
\hline & CG (58) & $90 \pm 23$ & - & - & $149 \pm 15$ & $62 \pm 11$ & $64 \pm 11$ & - & - & $146 \pm 22$ \\
\hline \multirow[t]{2}{*}{$\begin{array}{l}\text { Keener et al. } \\
\text { [63] }(2014,1)\end{array}$} & AG (61) & $83.2 \pm 11.5$ & $10.8 \pm 1.8$ & $\begin{array}{l}91.0 \pm \\
15.3\end{array}$ & $\begin{array}{l}164 \pm \\
13.4\end{array}$ & - & $62.0 \pm 16.4$ & - & - & - \\
\hline & CG (53) & $84.3 \pm 10.8$ & $10.6 \pm 2.5$ & $\begin{array}{l}93.3 \pm \\
10.6\end{array}$ & $\begin{array}{l}163 \pm \\
15.8\end{array}$ & - & $66.2 \pm 14.0$ & - & - & - \\
\hline \multirow[t]{2}{*}{$\begin{array}{l}\text { Kim et al. } \\
\text { [64] }(2012,1)\end{array}$} & AG (56) & $69.8 \pm 1.2$ & $9 \pm 0.9$ & $\begin{array}{l}73.3 \pm \\
8.7\end{array}$ & $\begin{array}{l}159.7 \pm \\
4.8\end{array}$ & - & $78.5 \pm 4$ & - & - & - \\
\hline & CG (49) & $69.9 \pm 2.3$ & $9 \pm 0.8$ & $\begin{array}{l}82.9 \pm \\
4.6\end{array}$ & $\begin{array}{l}153.7 \pm \\
3.9\end{array}$ & - & $81.3 \pm 6.1$ & - & - & - \\
\hline \multirow{2}{*}{$\begin{array}{l}\text { Klintberg } \\
\text { et al. [72] } \\
(2009, \text { II) }\end{array}$} & AG (7) & $74.2 \pm 12.9$ & - & - & $\begin{array}{l}146.3 \pm \\
18.8\end{array}$ & - & - & $50 \pm 8.7$ & $80 \pm 17.3$ & $160 \pm 20.2$ \\
\hline & CG (7) & $81 \pm 8.6$ & - & - & $150 \pm 8.7$ & - & - & $51.3 \pm 10.1$ & $61.3 \pm 21.6$ & $165 \pm 11.5$ \\
\hline \multirow[t]{2}{*}{$\begin{array}{l}\text { Koh et al. } \\
\text { [77] }(2014,1)\end{array}$} & AG (40) & $85.6 \pm 15.6$ & - & $\begin{array}{l}88.9 \pm \\
16.2\end{array}$ & $\begin{array}{l}143.7 \pm \\
23.5\end{array}$ & $9.1 \pm 2.9$ & $33.1 \pm 18.8$ & - & - & - \\
\hline & CG (48) & $88.7 \pm 9.7$ & $\therefore$ & $\begin{array}{l}92.1 \pm \\
10.2\end{array}$ & $\begin{array}{l}141.7 \pm \\
25.2\end{array}$ & $9.4 \pm 2.9$ & $29.5 \pm 21.2$ & - & - & - \\
\hline \multirow[t]{2}{*}{$\begin{array}{l}\text { Lee et al. } \\
\text { [67] }(2012, \text { II) }\end{array}$} & AG (30) & - & - & - & $\begin{array}{l}155.3 \pm \\
13.0\end{array}$ & - & $53.0 \pm 11.6$ & $65.7 \pm 13.3$ & $76.3 \pm 12.1$ & $\begin{array}{l}167.8 \pm \\
12.8\end{array}$ \\
\hline & CG (34) & - & - & - & $\begin{array}{l}153.0 \pm \\
12.2\end{array}$ & - & $48.1 \pm 13.9$ & $54.9 \pm 21.5$ & $77.7 \pm 11.6$ & $\begin{array}{l}161.8 \pm \\
27.3\end{array}$ \\
\hline \multirow{2}{*}{$\begin{array}{l}\text { Mazzocca } \\
\text { et al. [68] } \\
(2017, \text { II) }\end{array}$} & AG (31) & $82 \pm 15$ & $10.2 \pm 2.6$ & $\begin{array}{l}90 \pm \\
15\end{array}$ & $176 \pm 11$ & - & $61 \pm 18$ & - & - & - \\
\hline & CG (27) & $75 \pm 19$ & $9.3 \pm 3.6$ & $\begin{array}{l}84 \pm \\
19\end{array}$ & $173 \pm 17$ & - & $58 \pm 17$ & - & - & - \\
\hline \multirow{2}{*}{$\begin{array}{l}\text { Raschhofer } \\
\text { et al. [69] } \\
(2017, \text { II) }\end{array}$} & AG (14) & $78.8 \pm 1.4$ & - & - & - & - & - & - & - & - \\
\hline & CG (15) & $67 \pm 3.5$ & - & - & - & - & - & - & - & - \\
\hline \multirow[t]{2}{*}{$\begin{array}{l}\text { Sheps et al. } \\
\text { [78] }(2015, \text { II) }\end{array}$} & AG (97) & - & - & - & $\begin{array}{l}149.9 \pm \\
12.4\end{array}$ & - & - & $34.9 \pm 9.7$ & $81.5 \pm 11.7$ & $150.7 \pm 20$ \\
\hline & CG (92) & - & - & - & $149.9 \pm$ & - & - & $35.7 \pm 10.2$ & $84.0 \pm 11.9$ & $155.5 \pm$ \\
\hline
\end{tabular}


Table 2 Clinical outcomes and range of motions at a maximum of follow-up (Continued)

\begin{tabular}{|c|c|c|c|c|c|c|c|c|c|c|}
\hline \multirow{2}{*}{$\begin{array}{l}\text { Authors } \\
\text { (Year, level } \\
\text { of } \\
\text { evidence) }\end{array}$} & \multirow{2}{*}{$\begin{array}{l}\text { No of } \\
\text { patients in } \\
\text { the } \\
\text { accelerated } \\
\text { group (AG) } \\
\text { and } \\
\text { conservative } \\
\text { group (CG)a }\end{array}$} & \multicolumn{3}{|c|}{$\begin{array}{l}\text { Clinical outcomes } \\
\text { (Mean } \pm \text { standard deviation) }\end{array}$} & \multicolumn{6}{|c|}{$\begin{array}{l}\text { Range of motion } \\
\text { (Degrees } \pm \text { standard deviation) }\end{array}$} \\
\hline & & $\begin{array}{l}\text { Constant- } \\
\text { Murley } \\
\text { score }\end{array}$ & $\begin{array}{l}\text { Simple } \\
\text { shoulder } \\
\text { test }\end{array}$ & $\begin{array}{l}\text { ASES } \\
\text { score }\end{array}$ & $\begin{array}{l}\text { Forward } \\
\text { elevation }\end{array}$ & $\begin{array}{l}\text { Internal } \\
\text { rotation } \\
\text { at side }\end{array}$ & $\begin{array}{l}\text { External } \\
\text { rotation } \\
\text { at side }\end{array}$ & $\begin{array}{l}\text { Internal } \\
\text { rotation at } 90^{\circ} \\
\text { of abduction }\end{array}$ & $\begin{array}{l}\text { External } \\
\text { rotation at } 90^{\circ} \\
\text { of abduction }\end{array}$ & Abduction \\
\hline & & & & & 17.8 & & & & & 20.2 \\
\hline \multirow[t]{2}{*}{$\begin{array}{l}\text { Sheps et al. } \\
{[79](2019,1)}\end{array}$} & AG (103) & - & - & - & $\begin{array}{l}155.5 \pm \\
12.7\end{array}$ & - & - & $40.9 \pm 12$ & $76 \pm 14.8$ & $\begin{array}{l}153.5 \pm \\
14.6\end{array}$ \\
\hline & CG (103) & - & - & - & $\begin{array}{l}152.2 \pm \\
18.5\end{array}$ & - & - & $38.7 \pm 12.4$ & $71.5 \pm 17.8$ & $\begin{array}{l}152.2 \pm \\
21.9\end{array}$ \\
\hline \multirow[t]{2}{*}{$\begin{array}{l}\text { Tirefort et al. } \\
{[80](2019,1)}\end{array}$} & AG (40) & - & - & - & $\begin{array}{l}156.3 \pm \\
18.8\end{array}$ & - & - & - & $39.3 \pm 15.8$ & - \\
\hline & CG (40) & - & - & - & $\begin{array}{l}153.3 \pm \\
21.8\end{array}$ & - & - & - & $38.4 \pm 20.4$ & - \\
\hline
\end{tabular}

AG Accelerated Group, CG Conservative Group, $M$ Males, F Females, ASES American shoulder and elbow surgeons

${ }^{a}$ Data are reported as number of patients unless otherwise indicated

present study aims to compare the conservative and accelerated rehabilitation protocols in patients who underwent arthroscopic $\mathrm{RC}$ repair in terms of clinical outcomes and range of motions (ROMs) at 3, 6, 12, and 24-month follow-up.

\section{Materials and methods}

Search strategy and study selection

According to PRISMA 2020 guidelines, a systematic review was performed to evaluate accelerated or conservative rehabilitation's potential benefits in patients

Table 3 Retear rate

\begin{tabular}{|c|c|c|}
\hline $\begin{array}{l}\text { Authors } \\
\text { (Year, level of evidence) }\end{array}$ & No of patients in the accelerated group (AG) and conservative group (CG) & No of retear (\%) \\
\hline \multirow[t]{2}{*}{ Arndt et al. [61] (2012, II) } & AG (49) & $11(23.3 \%)$ \\
\hline & CG (43) & $7(15.4 \%)$ \\
\hline \multirow[t]{2}{*}{ Cuff et al. [70] $(2012,1)$} & AG (33) & $5(15 \%)$ \\
\hline & CG (35) & $3(8 \%)$ \\
\hline \multirow[t]{2}{*}{ De Roo et al. [110] $(2015, \mathrm{l})$} & AG (79) & $0(0 \%)$ \\
\hline & CG (51) & $2(4 \%)$ \\
\hline \multirow[t]{2}{*}{ Düzgün et al. [29] $(2014$, II) } & AG (19) & $0(0 \%)$ \\
\hline & CG (21) & $0(0 \%)$ \\
\hline \multirow[t]{2}{*}{ Keener et al. [63] $(2014$, I) } & AG (61) & $6(10 \%)$ \\
\hline & CG (53) & $3(6 \%)$ \\
\hline \multirow[t]{2}{*}{ Kim et al. [64] $(2012$, l) } & AG (56) & $7(12 \%)$ \\
\hline & CG (49) & $9(18 \%)$ \\
\hline \multirow[t]{2}{*}{ Koh et al. [77] $(2014,1)$} & AG (40) & $5(12.5 \%)$ \\
\hline & CG (48) & $4(8.3 \%)$ \\
\hline \multirow[t]{2}{*}{ Lee et al. [67] $(2012$, II) } & AG (30) & $7(23 \%)$ \\
\hline & CG (34) & $3(8.8 \%)$ \\
\hline \multirow[t]{2}{*}{ Mazzocca et al. [68] (2017, II) } & AG (31) & $11(34 \%)$ \\
\hline & CG (27) & $9(31 \%)$ \\
\hline \multirow[t]{2}{*}{ Sheps et al. [79] $(2019,1)$} & AG (103) & $5(4.9 \%)$ \\
\hline & CG (103) & 4 (3.9\%) \\
\hline
\end{tabular}


Table 4 Clinical outcomes and ROMs at 3-month follow-up

\begin{tabular}{|c|c|c|c|c|c|c|c|c|c|c|}
\hline \multirow{2}{*}{$\begin{array}{l}\text { Authors } \\
\text { (Year, level } \\
\text { of evidence) }\end{array}$} & \multirow{2}{*}{$\begin{array}{l}\text { No of } \\
\text { patients in } \\
\text { the } \\
\text { accelerated } \\
\text { group (AG) } \\
\text { and } \\
\text { conservative } \\
\text { group (CG) }^{\mathrm{a}}\end{array}$} & \multicolumn{3}{|c|}{$\begin{array}{l}\text { Clinical outcomes } \\
\text { (Mean } \pm \text { standard deviation) }\end{array}$} & \multicolumn{6}{|c|}{$\begin{array}{l}\text { Range of motion } \\
\text { (Degrees } \pm \text { standard deviation) }\end{array}$} \\
\hline & & $\begin{array}{l}\text { Constant- } \\
\text { Murley } \\
\text { score }\end{array}$ & $\begin{array}{l}\text { Simple } \\
\text { shoulder } \\
\text { test }\end{array}$ & $\begin{array}{l}\text { ASES } \\
\text { score }\end{array}$ & $\begin{array}{l}\text { Forward } \\
\text { elevation }\end{array}$ & $\begin{array}{l}\text { Internal } \\
\text { rotation at } \\
\text { side }\end{array}$ & $\begin{array}{l}\text { External } \\
\text { rotation at } \\
\text { side }\end{array}$ & $\begin{array}{l}\text { Internal } \\
\text { rotation in } \\
\text { abduction }\end{array}$ & $\begin{array}{l}\text { External } \\
\text { rotation in } \\
\text { abduction }\end{array}$ & Abduction \\
\hline \multirow[t]{2}{*}{$\begin{array}{l}\text { Arndt et al. } \\
\text { [61] }(2012, \text { II) }\end{array}$} & AG (49) & - & - & - & $\begin{array}{l}142.1 \pm \\
28.2\end{array}$ & - & $45.6 \pm 14.9$ & - & - & - \\
\hline & CG (43) & - & - & - & $\begin{array}{l}112.9 \pm \\
37.6\end{array}$ & - & $27.5 \pm 19.4$ & - & - & - \\
\hline \multirow[t]{2}{*}{$\begin{array}{l}\text { Düzgün et al. } \\
\text { [29] }(2014, \text { II) }\end{array}$} & AG (19) & - & - & - & $\begin{array}{l}154.4 \pm \\
2.2\end{array}$ & $80 \pm 3.7$ & $68.3 \pm 5.3$ & - & - & $166 \pm 5.9$ \\
\hline & CG (21) & - & - & - & $\begin{array}{l}141.4 \pm \\
5.9\end{array}$ & $68.2 \pm 3.3$ & $58.6 \pm 4.8$ & - & - & $151.9 \pm 5.3$ \\
\hline \multirow{2}{*}{$\begin{array}{l}\text { Jenssen et al. } \\
\text { [73] }(2018,1)\end{array}$} & AG (60) & $41 \pm 23$ & - & - & $112 \pm 31$ & $47 \pm 12$ & $45 \pm 19$ & - & - & $101 \pm 36$ \\
\hline & CG (58) & $38 \pm 19$ & - & - & $118 \pm 27$ & $48 \pm 12$ & $45 \pm 14$ & - & - & $102 \pm 32$ \\
\hline \multirow[t]{2}{*}{$\begin{array}{l}\text { Keener et al. } \\
{[63](2014,1)}\end{array}$} & AG (61) & - & - & - & $\begin{array}{l}136 \pm \\
23.6\end{array}$ & - & $47 \pm 18.5$ & - & - & \\
\hline & CG (53) & - & - & - & $\begin{array}{l}123 \pm \\
30.6\end{array}$ & - & $40.1 \pm 18.8$ & - & - & \\
\hline \multirow[t]{2}{*}{$\begin{array}{l}\text { Kim et al. [64] } \\
(2012, \mathrm{I})\end{array}$} & AG (56) & $63.2 \pm 1.7$ & $6.3 \pm 0.6$ & $\begin{array}{l}65.2 \pm \\
3\end{array}$ & $\begin{array}{l}144.9 \pm \\
2.8\end{array}$ & - & $71.2 \pm 4.5$ & - & - & - \\
\hline & CG (49) & $63.3 \pm 2.1$ & $6.1 \pm 0.7$ & $\begin{array}{l}64.7 \pm \\
3.5\end{array}$ & $140 \pm 3.9$ & - & $66,3 \pm 4.1$ & - & - & - \\
\hline \multirow[t]{2}{*}{$\begin{array}{l}\text { Lee et al. [67] } \\
(2012, \text { II) }\end{array}$} & AG (30) & - & - & - & $\begin{array}{l}149.7 \pm \\
12.7\end{array}$ & - & $44.2 \pm 14.6$ & $59 \pm 17.9$ & $70.5 \pm 14$ & $161.5 \pm 22$ \\
\hline & CG (34) & - & - & - & $\begin{array}{l}133.8 \pm \\
27.4\end{array}$ & - & $34.1 \pm 19.2$ & $38.5 \pm 24.1$ & $54 \pm 24.5$ & $\begin{array}{l}143.6 \pm \\
35.7\end{array}$ \\
\hline \multirow{2}{*}{$\begin{array}{l}\text { Mazzocca } \\
\text { et al. [68] } \\
(2017, \text { II) }\end{array}$} & AG (31) & $64 \pm 12$ & $7.4 \pm 2.4$ & $\begin{array}{l}70 \pm \\
13\end{array}$ & $168 \pm 14$ & - & $53 \pm 13$ & - & - & - \\
\hline & CG (27) & $61 \pm 15$ & $5.6 \pm 3.1$ & $\begin{array}{l}64 \pm \\
18\end{array}$ & $167 \pm 19$ & - & $55 \pm 19$ & - & - & - \\
\hline \multirow{2}{*}{$\begin{array}{l}\text { Raschhofer } \\
\text { et al. [69] } \\
(2017, \text { II) }\end{array}$} & AG (14) & $67.3 \pm 6.1$ & - & - & - & - & - & - & - & - \\
\hline & CG (15) & $58.3 \pm 2.6$ & - & - & - & - & - & - & - & - \\
\hline \multirow[t]{2}{*}{$\begin{array}{l}\text { Sheps et al. } \\
\text { [78] }(2015, \text { II) }\end{array}$} & AG (97) & - & - & - & $\begin{array}{l}120 \pm \\
25.4\end{array}$ & - & - & $23.7 \pm 16$ & $52.6 \pm 29.10$ & $\begin{array}{l}112.1 \pm \\
29.1\end{array}$ \\
\hline & CG (92) & - & - & - & $\begin{array}{l}118.5 \pm \\
25.5\end{array}$ & - & - & $24.1 \pm 13.8$ & $53.7 \pm 26.9$ & $113 \pm 26.20$ \\
\hline \multirow[t]{2}{*}{$\begin{array}{l}\text { Sheps et al. } \\
\text { [79] }(2019, \text { I) }\end{array}$} & AG (103) & - & - & - & $\begin{array}{l}125.5 \pm \\
28.5\end{array}$ & - & - & $30.2 \pm 15.2$ & $53 \pm 27.6$ & $\begin{array}{l}119.1 \pm \\
31.3\end{array}$ \\
\hline & CG (103) & - & - & - & $\begin{array}{l}121 \pm \\
30.1\end{array}$ & - & - & $25.9 \pm 16.3$ & $44.2 \pm 28.8$ & $116 \pm 37.6$ \\
\hline \multirow[t]{2}{*}{$\begin{array}{l}\text { Tirefort et al. } \\
{[80](2019,1)}\end{array}$} & AG (40) & - & - & - & $\begin{array}{l}125.8 \pm \\
24.4\end{array}$ & - & $27.5 \pm 18$ & - & - & - \\
\hline & CG (40) & - & - & - & $\begin{array}{l}153.3 \pm \\
21.8\end{array}$ & - & $34.1 \pm 17.8$ & - & - & - \\
\hline
\end{tabular}

AG Accelerated Group, CG Conservative Group, ASES American shoulder and elbow surgeons ${ }^{a}$ Data are reported as number of patients unless otherwise indicated 
Table 5 Clinical outcomes and ROMs at 6-month follow-up

\begin{tabular}{|c|c|c|c|c|c|c|c|c|c|c|}
\hline \multirow{2}{*}{$\begin{array}{l}\text { Authors } \\
\text { (Year, level } \\
\text { of evidence) }\end{array}$} & \multirow{2}{*}{$\begin{array}{l}\text { No of } \\
\text { patients in } \\
\text { the } \\
\text { accelerated } \\
\text { group (AG) } \\
\text { and } \\
\text { conservative }_{\text {group (CG) }}\end{array}$} & \multicolumn{3}{|c|}{$\begin{array}{l}\text { Clinical outcomes } \\
\text { (Mean } \pm \text { standard deviation) }\end{array}$} & \multicolumn{6}{|c|}{$\begin{array}{l}\text { Range of motion } \\
\text { (Degrees } \pm \text { standard deviation) }\end{array}$} \\
\hline & & $\begin{array}{l}\text { Constant- } \\
\text { Murley } \\
\text { score }\end{array}$ & $\begin{array}{l}\text { Simple } \\
\text { shoulder } \\
\text { test }\end{array}$ & $\begin{array}{l}\text { ASES } \\
\text { score }\end{array}$ & $\begin{array}{l}\text { Forward } \\
\text { elevation }\end{array}$ & $\begin{array}{l}\text { Internal } \\
\text { rotation at } \\
\text { side }\end{array}$ & $\begin{array}{l}\text { External } \\
\text { rotation at } \\
\text { side }\end{array}$ & $\begin{array}{l}\text { Internal } \\
\text { rotation in } \\
\text { abduction }\end{array}$ & $\begin{array}{l}\text { External } \\
\text { rotation in } \\
\text { abduction }\end{array}$ & Abduction \\
\hline \multirow{2}{*}{$\begin{array}{l}\text { Arndt et al. } \\
{[61]} \\
(2012, \text { II) }\end{array}$} & AG (49) & - & - & - & $\begin{array}{l}158.4 \pm \\
22.9\end{array}$ & - & $54.3 \pm 12.5$ & - & - & - \\
\hline & CG (43) & - & - & - & $\begin{array}{l}146.4 \pm \\
30\end{array}$ & - & $44.3 \pm 19.4$ & - & - & - \\
\hline \multirow{2}{*}{$\begin{array}{l}\text { Cuff et al. } \\
{[70]} \\
(2012, I)\end{array}$} & AG (33) & - & - & - & 172 & 79 & 44 & - & - & - \\
\hline & CG (35) & - & - & - & 165 & 60 & 43 & - & - & - \\
\hline \multirow{2}{*}{$\begin{array}{l}\text { Düzgün et al. } \\
{[71]} \\
(2011, \text { II) }\end{array}$} & AG (13) & - & - & - & - & - & - & - & - & - \\
\hline & CG (16) & - & - & - & - & - & - & - & - & - \\
\hline \multirow{2}{*}{$\begin{array}{l}\text { Düzgün et al. } \\
\text { [29] } \\
(2014, \text { II) }\end{array}$} & AG (19) & - & - & - & $\begin{array}{l}158.9 \pm \\
1.8\end{array}$ & $88.2 \pm 2.1$ & $86.6 \pm 2.4$ & - & - & $173.6 \pm 3.4$ \\
\hline & CG (21) & - & - & - & $153.7 \pm 4$ & $86.5 \pm 1.9$ & $84.1 \pm 2.2$ & - & - & $171 \pm 3.1$ \\
\hline \multirow{2}{*}{$\begin{array}{l}\text { Jenssen et al. } \\
{[73]} \\
(2018, \mathrm{I})\end{array}$} & AG (60) & $68 \pm 28$ & - & - & $138 \pm 25$ & $56 \pm 10$ & $57 \pm 15$ & - & - & $130 \pm 33$ \\
\hline & CG (58) & $71 \pm 25$ & - & - & $141 \pm 20$ & $56 \pm 14$ & $59 \pm 15$ & - & - & $134 \pm 30$ \\
\hline \multirow{2}{*}{$\begin{array}{l}\text { Keener et al. } \\
{[63]} \\
(2014,1)\end{array}$} & AG (61) & $74.4 \pm 13.3$ & $9.1 \pm 2.7$ & $\begin{array}{l}81.1 \pm \\
16.2\end{array}$ & $\begin{array}{l}155 \pm \\
18.1\end{array}$ & - & $61.6 \pm 17.8$ & - & - & $80 \pm 14.10$ \\
\hline & CG (53) & $74.6 \pm 11.3$ & $9.3 \pm 2.9$ & $\begin{array}{l}84.3 \pm \\
15.1\end{array}$ & $\begin{array}{l}154 \pm \\
17.8\end{array}$ & - & $63.9 \pm 15.1$ & - & - & $81.3 \pm 13$ \\
\hline \multirow[t]{2}{*}{$\begin{array}{l}\text { Kim et al. [64] } \\
(2012, \text { I) }\end{array}$} & AG (56) & $66.1 \pm 1.7$ & $7.8 \pm 0.5$ & $\begin{array}{l}67.1 \pm \\
3.1\end{array}$ & $\begin{array}{l}150.6 \pm \\
5.1\end{array}$ & - & $77.2 \pm 3.1$ & - & - & - \\
\hline & CG (49) & $64.5 \pm 2.1$ & $6.7 \pm 0.6$ & $\begin{array}{l}69.9 \pm \\
3.3\end{array}$ & $\begin{array}{l}147.1 \pm \\
3.5\end{array}$ & - & $72.9 \pm 4.9$ & - & - & - \\
\hline \multirow{2}{*}{$\begin{array}{l}\text { Klintberg } \\
\text { et al. [72] } \\
(2009, \text { II) }\end{array}$} & AG (7) & $63.5 \pm 9.8$ & - & - & $\begin{array}{l}138.8 \pm \\
15.9\end{array}$ & - & - & $49 \pm 14.4$ & $63.8 \pm 21.6$ & $157.75 \pm 13$ \\
\hline & CG (7) & $64.8 \pm 18.7$ & - & - & $\begin{array}{l}126.3 \pm \\
30.3\end{array}$ & - & - & $41.3 \pm 7.2$ & $70 \pm 17.3$ & $\begin{array}{l}142.5 \pm \\
37.5\end{array}$ \\
\hline \multirow[t]{2}{*}{$\begin{array}{l}\text { Lee et al. [67] } \\
(2012, \text { II) }\end{array}$} & AG (30) & - & - & - & $\begin{array}{l}157.3 \pm \\
11.4\end{array}$ & - & $50.3 \pm 11.2$ & $63.8 \pm 14.3$ & $74 \pm 11$ & $\begin{array}{l}165.3 \pm \\
13.9\end{array}$ \\
\hline & CG (34) & - & - & - & $\begin{array}{l}151.9 \pm \\
18.2\end{array}$ & - & $41.6 \pm 14.9$ & $47.3 \pm 22.7$ & $67.8 \pm 18.1$ & $\begin{array}{l}154.4 \pm \\
30.1\end{array}$ \\
\hline \multirow{2}{*}{$\begin{array}{l}\text { Mazzocca } \\
\text { et al. [68] } \\
(2017, \text { II) }\end{array}$} & AG (31) & $78 \pm 11$ & $10 \pm 2.4$ & $\begin{array}{l}88 \pm \\
16\end{array}$ & $173 \pm 20$ & - & $63 \pm 16$ & - & - & - \\
\hline & CG (27) & $75 \pm 15$ & $8.7 \pm 3.3$ & $\begin{array}{l}80 \pm \\
19\end{array}$ & $173 \pm 10$ & - & $61 \pm 17$ & - & - & - \\
\hline \multirow{2}{*}{$\begin{array}{l}\text { Raschhofer } \\
\text { et al. [69] } \\
(2017, \text { II) }\end{array}$} & AG (14) & $78.8 \pm 1.4$ & - & - & - & - & - & - & - & - \\
\hline & CG (15) & $67 \pm 3.5$ & - & - & - & - & - & - & - & - \\
\hline \multirow{2}{*}{$\begin{array}{l}\text { Sheps et al. } \\
{[78]} \\
(2015, \|)\end{array}$} & AG (97) & - & - & - & $\begin{array}{l}136.3 \pm \\
18.1\end{array}$ & - & - & $32.2 \pm 12.7$ & $73.1 \pm 17.3$ & $\begin{array}{l}134.6 \pm \\
27.3\end{array}$ \\
\hline & CG (92) & - & - & - & $\begin{array}{l}135.9 \pm \\
21.6\end{array}$ & - & - & $32.9 \pm 12$ & $74.2 \pm 17.1$ & $\begin{array}{l}139.1 \pm \\
25.8\end{array}$ \\
\hline \multirow{2}{*}{$\begin{array}{l}\text { Sheps et al. } \\
{[79]} \\
(2019,1)\end{array}$} & AG (103) & - & - & - & $\begin{array}{l}142.3 \pm \\
22.6\end{array}$ & - & - & $35.9 \pm 12.1$ & $67.3 \pm 21$ & $\begin{array}{l}139.4 \pm \\
23.5\end{array}$ \\
\hline & CG (103) & - & - & - & $\begin{array}{l}141.3 \pm \\
22.6\end{array}$ & - & - & $35.5 \pm 12.2$ & $62.9 \pm 21.6$ & $\begin{array}{l}139.5 \pm \\
27.60\end{array}$ \\
\hline $\begin{array}{l}\text { Tirefort et al. } \\
{[80]}\end{array}$ & AG (40) & - & - & - & $\begin{array}{l}156.3 \pm \\
18.8\end{array}$ & - & $39.3 \pm 15.8$ & - & - & - \\
\hline
\end{tabular}


Table 5 Clinical outcomes and ROMs at 6-month follow-up (Continued)

\begin{tabular}{|c|c|c|c|c|c|c|c|c|c|c|}
\hline \multirow{2}{*}{$\begin{array}{l}\text { Authors } \\
\text { (Year, level } \\
\text { of evidence) }\end{array}$} & \multirow{2}{*}{$\begin{array}{l}\text { No of } \\
\text { patients in } \\
\text { the } \\
\text { accelerated } \\
\text { group (AG) } \\
\text { and } \\
\text { conservative } \\
\text { group (CG) }^{\mathrm{a}}\end{array}$} & \multicolumn{3}{|c|}{$\begin{array}{l}\text { Clinical outcomes } \\
\text { (Mean } \pm \text { standard deviation) }\end{array}$} & \multicolumn{6}{|c|}{$\begin{array}{l}\text { Range of motion } \\
\text { (Degrees } \pm \text { standard deviation) }\end{array}$} \\
\hline & & $\begin{array}{l}\text { Constant- } \\
\text { Murley } \\
\text { score }\end{array}$ & $\begin{array}{l}\text { Simple } \\
\text { shoulder } \\
\text { test }\end{array}$ & $\begin{array}{l}\text { ASES } \\
\text { score }\end{array}$ & $\begin{array}{l}\text { Forward } \\
\text { elevation }\end{array}$ & $\begin{array}{l}\text { Internal } \\
\text { rotation at } \\
\text { side }\end{array}$ & $\begin{array}{l}\text { External } \\
\text { rotation at } \\
\text { side }\end{array}$ & $\begin{array}{l}\text { Internal } \\
\text { rotation in } \\
\text { abduction }\end{array}$ & $\begin{array}{l}\text { External } \\
\text { rotation in } \\
\text { abduction }\end{array}$ & Abduction \\
\hline$(2019,1)$ & CG (40) & - & - & - & $\begin{array}{l}153.3 \pm \\
21.8\end{array}$ & - & $38.4 \pm 20.4$ & - & - & - \\
\hline
\end{tabular}

AG Accelerated Group, CG Conservative Group, ASES American shoulder and elbow surgeons

${ }^{a}$ Data are reported as number of patients unless otherwise indicated

undergoing arthroscopic RC repair [99]. Conservative rehabilitation has been defined as a complete immobilization, whereas accelerated rehabilitation gradually reintroduces movements before the sixth week. The analysis has been executed since the beginning of the Cochrane Central Register of Controlled Trials (CENTRAL), MEDLINE, EMBASE, and Google scholar databases until March 31, 2020. The combination of free-text terms and Medical Subject Headings $(\mathrm{MeSH})$ in the title and abstract was used to perform the research. The search strategy was built on the application of Boolean logic operators to the following keywords: ("rotator cuff" OR "rotator cuff tear" OR "rotator cuff repair") AND (rehabilitation OR "postoperative rehabilitation" OR exercises OR "physical therapy" OR "physical therapies" OR "rehabilitation protocol" OR "rehabilitation program" OR "accelerated rehabilitation" OR "early rehabilitation" OR "conservative rehabilitation" OR "delayed rehabilitation" OR "slow rehabilitation"). Three independent reviewers (U.G.L., L.R.A. and V.D.) had verified the suitability of each article published in a peer-reviewed journal for the relevance of title and abstract to the objective of this study without excluding any journal. Records also include the listed references from the original record as another possible source of relevant trial reports. To increase the study's strength, only level I-II studies based on the Oxford Centre of EBM were selected and involved in the present research [100]. Studies without abstract or meaningful information were excluded during the study selection process. The independent reviewers conducted an accurate full-text reading of the chosen articles, obtaining data to reduce selection bias.

To be included in this review, eligible studies had to meet the following inclusion criteria:

(i) A comparison between conservative and accelerated rehabilitation protocol; (ii) A primary arthroscopic $\mathrm{RC}$ repair must be performed in patients of both study groups;

(iii) Reported at least one of retear rates, clinical scores, and range of motions;

(iv) Level I-II articles published in a peer-reviewed magazine or presented at a conference.

Exclusion criteria were:

(i) A comparison between one of the two forms of rehabilitation and healthy control;

(ii) Other RC repairs (i.e. tendon reconstruction, arthroplasty, tendon transfer or revision);

(iii) Lack of enough data for extraction;

(iv) Reviews, case reports, articles on animals, cadaver, or in vitro researches, biomechanical reports, technical notes, letters, and instructional studies.

\section{Data extraction process}

For each article included in the study, the following data has been extracted: authors, year, study design, level of evidence, sample size both at baseline and at final follow-up, losses at follow-up, number of patients in the accelerated and conservative group, sex, age, follow-up, clinical outcomes (Constant-Murley score (CMS), Simple Shoulder Test (SST) score, and American Shoulder and elbow surgeons (ASES) score), ROMs (forward flexion, internal rotation, external rotation, internal rotation in abduction, external rotation in abduction, abduction), visual analogue scale (VAS) score, retear events, and exercises performed during the rehabilitation period for both groups.

\section{Quality assessment}

Two independent reviewers (L.R.A and U.G.L.) assessed the risk of bias for each included study. Review Manager (RevMan, version 5 for Windows; Cochrane Information Management System) was used as risk of bias 
Table 6 Clinical outcomes and ROMs at 12-month follow-up

\begin{tabular}{|c|c|c|c|c|c|c|c|c|c|c|}
\hline \multirow{2}{*}{$\begin{array}{l}\text { Authors } \\
\text { (Year, level } \\
\text { of evidence) }\end{array}$} & \multirow{2}{*}{$\begin{array}{l}\text { No of } \\
\text { patients in } \\
\text { the } \\
\text { accelerated } \\
\text { group (AG) } \\
\text { and } \\
\text { conservative } \\
\text { group (CG) }\end{array}$} & \multicolumn{3}{|c|}{$\begin{array}{l}\text { Clinical outcomes } \\
\text { (Mean } \pm \text { standard deviation) }\end{array}$} & \multicolumn{6}{|c|}{$\begin{array}{l}\text { Range of motion } \\
\text { (Degrees } \pm \text { standard deviation) }\end{array}$} \\
\hline & & $\begin{array}{l}\text { Constant- } \\
\text { Murley } \\
\text { score }\end{array}$ & $\begin{array}{l}\text { Simple } \\
\text { shoulder } \\
\text { test }\end{array}$ & $\begin{array}{l}\text { ASES } \\
\text { score }\end{array}$ & $\begin{array}{l}\text { Forward } \\
\text { elevation }\end{array}$ & $\begin{array}{l}\text { Internal } \\
\text { rotation at } \\
\text { side }\end{array}$ & $\begin{array}{l}\text { External } \\
\text { rotation at } \\
\text { side }\end{array}$ & $\begin{array}{l}\text { Internal } \\
\text { rotation in } \\
\text { abduction }\end{array}$ & $\begin{array}{l}\text { External } \\
\text { rotation in } \\
\text { abduction }\end{array}$ & Abduction \\
\hline \multirow{2}{*}{$\begin{array}{l}\text { Arndt et al. } \\
{[61]} \\
(2012, \text { II) }\end{array}$} & AG (49) & - & - & - & $\begin{array}{l}171.9 \pm \\
13.6\end{array}$ & - & $58.1 \pm 13.2$ & - & - & - \\
\hline & CG (43) & - & - & - & $\begin{array}{l}161.9 \pm \\
26.2\end{array}$ & - & $48.3 \pm 18.2$ & - & - & - \\
\hline \multirow{2}{*}{$\begin{array}{l}\text { Cuff et al. } \\
{[70]} \\
(2012,1)\end{array}$} & AG (33) & - & 11.01 & 91.1 & 174 & 94 & 46 & - & - & - \\
\hline & CG (35) & - & 11.01 & 92.8 & 173 & 91 & 45 & - & - & - \\
\hline \multirow{2}{*}{$\begin{array}{l}\text { Jenssen et al. } \\
{[73]} \\
(2018,1)\end{array}$} & AG (60) & $86 \pm 27$ & - & - & $151 \pm 15$ & $61 \pm 9$ & $64 \pm 12$ & - & - & $146 \pm 22$ \\
\hline & CG (58) & $90 \pm 23$ & - & - & $149 \pm 15$ & $62 \pm 11$ & $64 \pm 11$ & - & - & $146 \pm 22$ \\
\hline \multirow{2}{*}{$\begin{array}{l}\text { Keener et al. } \\
{[63]} \\
(2014, \mathrm{l})\end{array}$} & $A G(61)$ & $79.1 \pm 10$ & $10.3 \pm 2.3$ & $\begin{array}{l}88.1 \pm \\
15.8\end{array}$ & $\begin{array}{l}161 \pm \\
13.4\end{array}$ & - & $64.1 \pm 15.2$ & - & - & $84.7 \pm 13.9$ \\
\hline & CG (53) & $79.9 \pm 12.3$ & $10 \pm 3.1$ & $\begin{array}{l}89.1 \pm \\
14.1\end{array}$ & $\begin{array}{l}159 \pm \\
22.8\end{array}$ & - & $67.3 \pm 15.9$ & - & - & $88.6 \pm 11.9$ \\
\hline \multirow{2}{*}{$\begin{array}{l}\text { Kim et al. } \\
{[64]} \\
(2012,1)\end{array}$} & AG (56) & $69.8 \pm 1.2$ & $9 \pm 0.9$ & $\begin{array}{l}73.3 \pm \\
8.7\end{array}$ & $\begin{array}{l}159.7 \pm \\
4.8\end{array}$ & - & $78.5 \pm 4$ & - & - & - \\
\hline & CG (49) & $69.9 \pm 2.3$ & $9 \pm 0.8$ & $\begin{array}{l}82.9 \pm \\
4.6\end{array}$ & $\begin{array}{l}153.7 \pm \\
3.9\end{array}$ & - & $81.3 \pm 6.1$ & - & - & - \\
\hline \multirow{2}{*}{$\begin{array}{l}\text { Klintberg } \\
\text { et al. [72] } \\
(2009, \text { II) }\end{array}$} & $A G(7)$ & $81 \pm 8.6$ & - & - & $\begin{array}{l}146.3 \pm \\
18.8\end{array}$ & - & - & $50 \pm 8.7$ & $80 \pm 17.3$ & $160 \pm 20.2$ \\
\hline & CG (7) & $74.3 \pm 13$ & - & - & $150 \pm 8.7$ & - & - & $51.3 \pm 10.1$ & $61.3 \pm 21.6$ & $165 \pm 11.5$ \\
\hline \multirow{2}{*}{$\begin{array}{l}\text { Lee et al. } \\
{[67]} \\
(2012, \text { II) }\end{array}$} & AG (30) & - & - & - & $\begin{array}{l}155.3 \pm \\
13\end{array}$ & - & $53 \pm 11.6$ & $65.7 \pm 13.3$ & $76.3 \pm 12.1$ & $\begin{array}{l}167.8 \pm \\
12.8\end{array}$ \\
\hline & CG (34) & - & - & - & $\begin{array}{l}153 \pm \\
12.2\end{array}$ & - & $48.1 \pm 13.9$ & $54.9 \pm 21.5$ & $77.7 \pm 11.6$ & $\begin{array}{l}161.8 \pm \\
27.3\end{array}$ \\
\hline \multirow{2}{*}{$\begin{array}{l}\text { Mazzocca } \\
\text { et al. [68] } \\
(2017, \text { II) }\end{array}$} & AG (31) & $82 \pm 15$ & $10.2 \pm 2.6$ & $\begin{array}{l}90 \pm \\
15\end{array}$ & $176 \pm 11$ & - & $61 \pm 18$ & - & - & - \\
\hline & CG (27) & $75 \pm 19$ & $9.3 \pm 3.6$ & $\begin{array}{l}84 \pm \\
19\end{array}$ & $173 \pm 17$ & - & $58 \pm 17$ & - & - & - \\
\hline \multirow{2}{*}{$\begin{array}{l}\text { Sheps et al. } \\
{[78]} \\
(2015, \text { II) }\end{array}$} & AG (97) & - & - & - & $\begin{array}{l}144.8 \pm \\
14.4\end{array}$ & - & - & $33.9 \pm 9.9$ & $77.9 \pm 13.4$ & $\begin{array}{l}144.7 \pm \\
22.6\end{array}$ \\
\hline & CG (92) & - & - & - & $\begin{array}{l}114.8 \pm \\
19.2\end{array}$ & - & - & $34.8 \pm 10.3$ & $80.3 \pm 13.9$ & $\begin{array}{l}149.9 \pm \\
23.2\end{array}$ \\
\hline \multirow{2}{*}{$\begin{array}{l}\text { Sheps et al. } \\
{[79]} \\
(2019,1)\end{array}$} & AG (103) & - & - & - & $\begin{array}{l}150.9 \pm \\
12.6\end{array}$ & - & - & $39 \pm 12.4$ & $75.3 \pm 16.2$ & $150 \pm 15.3$ \\
\hline & CG (103) & - & - & - & $\begin{array}{l}149 \pm \\
19.9\end{array}$ & - & - & $40.1 \pm 14.4$ & $70.6 \pm 18.8$ & $\begin{array}{l}148.4 \pm \\
22.4\end{array}$ \\
\hline
\end{tabular}

AG Accelerated Group, CG Conservative Group, ASES American shoulder and elbow surgeons

${ }^{a}$ Data are reported as number of patients unless otherwise indicated

assessment tool. Following methods recommended by The Cochrane Collaboration, a domain-based evaluation (random sequence generation; allocation concealment; blinding of participants, personnel and outcome assessors; incomplete outcome data; selective outcome data reporting and other sources of bias) was performed [101]. The following judgments were used: low risk, high risk, or unclear (either lack of information or uncertainty over the potential for bias). Kappa statistics were used to assess inter-rater reliability between data extraction and quality assessment [102].

The GRADE (Grading of Recommendations Assessment, Development and Evaluation) guidelines were used to assess the critical appraisal status and quality 
Table 7 Clinical outcomes and ROMs at 24-month follow-up

\begin{tabular}{|c|c|c|c|c|c|c|c|c|c|}
\hline \multirow{2}{*}{$\begin{array}{l}\text { Authors } \\
\text { (Year, level } \\
\text { of evidence) }\end{array}$} & \multirow{2}{*}{$\begin{array}{l}\text { No of } \\
\text { patients in } \\
\text { the } \\
\text { accelerated } \\
\text { group (AG) } \\
\text { and } \\
\text { conservative } \\
\text { group (CG) }\end{array}$} & \multicolumn{3}{|c|}{$\begin{array}{l}\text { Clinical outcomes } \\
\text { (Mean } \pm \text { standard deviation) }\end{array}$} & \multicolumn{5}{|c|}{$\begin{array}{l}\text { Range of motion } \\
\text { (Degrees } \pm \text { standard deviation) }\end{array}$} \\
\hline & & $\begin{array}{l}\text { Constant- } \\
\text { Murley } \\
\text { score }\end{array}$ & $\begin{array}{l}\text { Simple } \\
\text { shoulder } \\
\text { test }\end{array}$ & $\begin{array}{l}\text { ASES } \\
\text { score }\end{array}$ & $\begin{array}{l}\text { Forward } \\
\text { elevation }\end{array}$ & $\begin{array}{l}\text { External } \\
\text { rotation at } \\
\text { side }\end{array}$ & $\begin{array}{l}\text { Internal rotation } \\
\text { at } 90^{\circ} \text { of } \\
\text { abduction }\end{array}$ & $\begin{array}{l}\text { External rotation } \\
\text { at } 90^{\circ} \text { of } \\
\text { abduction }\end{array}$ & Abduction \\
\hline \multirow{2}{*}{$\begin{array}{l}\text { Keener et al. } \\
{[63]} \\
(2014,1)\end{array}$} & AG (61) & $83.2 \pm 11.5$ & $10.8 \pm 1.8$ & $\begin{array}{l}91 \pm \\
15.3\end{array}$ & $164 \pm 13.4$ & $62 \pm 16.4$ & - & - & $90 \pm 10.3$ \\
\hline & CG (53) & $84.3 \pm 10.8$ & $10.6 \pm 2.5$ & $\begin{array}{l}93.3 \pm \\
10.6\end{array}$ & $163 \pm 15.8$ & $66.2 \pm 14$ & - & - & $87.7 \pm 11.9$ \\
\hline \multirow{2}{*}{$\begin{array}{l}\text { Klintberg } \\
\text { et al. [72] } \\
(2009, \text { II) }\end{array}$} & AG (7) & $82.3 \pm 6.1$ & - & - & - & - & - & - & - \\
\hline & CG (7) & $75.8 \pm 11.8$ & - & - & - & - & - & - & - \\
\hline \multirow{2}{*}{$\begin{array}{l}\text { Sheps et al. } \\
{[78]} \\
(2015, \text { II) }\end{array}$} & AG (97) & - & - & - & $\begin{array}{l}149.9 \pm \\
12.4\end{array}$ & - & $34.9 \pm 9.7$ & $81.5 \pm 11.7$ & $150.7 \pm 20$ \\
\hline & CG (92) & - & - & - & $\begin{array}{l}149.9 \pm \\
17.8\end{array}$ & - & $35.7 \pm 10.2$ & $84 \pm 11.9$ & $\begin{array}{l}155.5 \pm \\
20.2\end{array}$ \\
\hline \multirow{2}{*}{$\begin{array}{l}\text { Sheps et al. } \\
{[79]} \\
(2019, \text { I) }\end{array}$} & AG (103) & - & - & - & $\begin{array}{l}155.5 \pm \\
12.7\end{array}$ & - & $40.9 \pm 12$ & $76 \pm 14.8$ & $\begin{array}{l}153.5 \pm \\
14.6\end{array}$ \\
\hline & CG (103) & - & - & - & $\begin{array}{l}152.2 \pm \\
18.5\end{array}$ & - & $38.7 \pm 12.4$ & $71.5 \pm 17.8$ & $\begin{array}{l}152.2 \pm \\
21.9\end{array}$ \\
\hline
\end{tabular}

AG Accelerated Group, CG Conservative Group, ASES American shoulder and elbow surgeons

${ }^{a}$ Data are reported as number of patients unless otherwise indicated

of evidence of the included randomised controlled trials. The combination of four factors (i.e., study design, study quality, consistency, and directness) provided whether the evidence's quality was high, moderate, low, or very low. We downgraded the evidence quality from' high quality' by one level for serious risk of bias, inconsistency, indirectness of evidence, imprecision of effect estimates or potential publication bias [103].

\section{Meta-analysis}

A meta-analysis was performed of all included studies to compare accelerated versus conservative rehabilitation protocols after arthroscopic RC repair. The retear rate, postoperative CMS and ROMs at 6, 12, and 24 months of follow-up were the outcomes measured. Review Manager (RevMan, version 5 for Windows; Cochrane Information Management System) was used to determine the treatment effect's magnitude. An $\mathrm{I}^{2}$ index evaluated the heterogeneity of the principal analysis. $\mathrm{I}^{2}$ index describes the percentage of the whole diversity between studies that are made by heterogeneity. According to the Cochrane Handbook for Systematic Reviews of Interventions, the interpretation of the $\mathrm{I}^{2}$ for heterogeneity was as follows:

- 0-40\%, was not important.

- 30-60\%, represented moderate heterogeneity.
- $50-90 \%$, represented substantial heterogeneity.

- $\quad 75-100 \%$, represented considerable heterogeneity.

A fixed-effect model in the data synthesis was adopted when heterogeneity values were $\leq 60 \%$; otherwise, a random-effects model was used.

Continuous variables were reported as mean \pm standard deviation, rounded to a decimal plane. In all studies, $P<0.05$ was considered statistically significant. For each statistically significant outcome in terms of CMS and ROMs, the achievement of the minimal clinically important difference (MCID) between the two groups was evaluated to determine clinical reliability.

\section{Results}

\section{Search results}

The research strategy has comprehensively yielded 2393 results. After removing duplicates, 1885 articles were filtered by the study design (i.e. level I-II clinical studies). Among the selected 25 clinical studies, four articles were excluded because they compared passive ROM exercises and continuous passive movement [104-107]. Another four clinical studies were excluded because they compared a supervised and selfassisted rehabilitation protocol[55-57, 108]. Conservative rehabilitation was defined as complete immobilization, whereas accelerated rehabilitation was a gradual reintroduction of movement before week 6 . 
Table 8 VAS score at 3, 6, 12, and 24-month follow-up

\begin{tabular}{|c|c|c|c|c|c|}
\hline \multirow{2}{*}{$\begin{array}{l}\text { Authors } \\
\text { (Year, level of evidence) }\end{array}$} & \multirow[b]{2}{*}{$\begin{array}{l}\text { No of } \\
\text { patients in } \\
\text { the } \\
\text { accelerated } \\
\text { group (AG) } \\
\text { and } \\
\text { conservative }_{\text {group (CG) }}\end{array}$} & \multicolumn{4}{|c|}{ VAS score (mean \pm standard deviation) } \\
\hline & & 3-month follow-up & 6-month follow-up & 12-month follow-up & 24-month follow-up \\
\hline \multirow[t]{2}{*}{ Jenssen et al. [73] $(2018, \mathrm{I})$} & AG (60) & - & - & $8.6 \pm 1.8$ & - \\
\hline & CG (58) & - & - & $8.7 \pm 1.9$ & - \\
\hline \multirow[t]{2}{*}{ Keener et al. [63] $(2014$, I) } & $A G(61)$ & - & $1.4 \pm 1.6$ & $1.1 \pm 1.7$ & $0.9 \pm 1.7$ \\
\hline & CG (53) & - & $1.1 \pm 1.4$ & $0.9 \pm 1.2$ & $0.6 \pm 1.1$ \\
\hline \multirow[t]{2}{*}{ Kim et al. [64] $(2012,1)$} & AG (56) & - & 3 & 2.8 & - \\
\hline & CG (49) & - & 3.2 & 1.8 & - \\
\hline \multirow[t]{2}{*}{ Klintberg et al. [72] (2009, II) } & $A G(7)$ & $2.5 \pm 0.5$ & $1.2 \pm 0.8$ & 0 & $0.8 \pm 0.9$ \\
\hline & CG (7) & $2.4 \pm 1.7$ & $0.3 \pm 0.5$ & $0.9 \pm 1.1$ & $0.3 \pm 0.4$ \\
\hline \multirow[t]{2}{*}{ Koh et al. [77] $(2014, \mathrm{l})$} & AG (40) & - & $2.7 \pm 1.5$ & - & $1.3 \pm 1.8$ \\
\hline & CG (48) & - & $2.7 \pm 1.8$ & - & $0.8 \pm 1.0$ \\
\hline \multirow[t]{2}{*}{ Lee et al. [67] (2012, II) } & AG (30) & - & - & $0.9 \pm 0.9$ & - \\
\hline & CG (34) & - & - & $0.8 \pm 0.9$ & - \\
\hline \multirow[t]{2}{*}{ Mazzocca et al. [68] (2017, II) } & AG (31) & $1.6 \pm 1.4$ & $1.0 \pm 1.7$ & $0.7 \pm 1.5$ & - \\
\hline & CG (27) & $2.7 \pm 2.3$ & $1.4 \pm 2.3$ & $0.9 \pm 1.5$ & - \\
\hline \multirow[t]{2}{*}{ Raschhofer et al. [69] (2017, II) } & AG (14) & $1.9 \pm 0.7$ & $0.4 \pm 0.4$ & - & - \\
\hline & CG (15) & $2 \pm 0.8$ & $0.1 \pm 0.5$ & - & - \\
\hline \multirow[t]{2}{*}{ Sheps et al. [78] $(2015$, II) } & AG (97) & $1.4 \pm 1.6$ & $0.8 \pm 1.3$ & $0.5 \pm 0.8$ & $0.4 \pm 0.9$ \\
\hline & CG (92) & $1.3 \pm 1.6$ & $0.9 \pm 1.6$ & $0.9 \pm 1.7$ & $0.5 \pm 1.3$ \\
\hline \multirow[t]{2}{*}{ Sheps et al. [79] $(2019$, I) } & AG (103) & $1.8 \pm 2.1$ & $0.9 \pm 1.3$ & $0.6 \pm 1.0$ & $0.7 \pm 1.3$ \\
\hline & CG (103) & $1.4 \pm 1.6$ & $0.8 \pm 1.0$ & $0.6 \pm 1.0$ & $0.6 \pm 1.3$ \\
\hline \multirow[t]{2}{*}{ Tirefort et al. [80] $(2019, \mathrm{I})$} & AG (40) & $1.9 \pm 1.9$ & $0.8 \pm 1.1$ & - & - \\
\hline & CG (40) & $2.6 \pm 2.1$ & $1.5 \pm 1.6$ & - & - \\
\hline
\end{tabular}

AG Accelerated Group, CG Conservative Group, ASES American shoulder and elbow surgeons

${ }^{a}$ Data are reported as number of patients unless otherwise indicated

Therefore, one study was excluded because it did not fulfill these definitions [109]. Hence, at the end of the thorough investigation by the reviewers, 16 of the 25 studies met the inclusion criteria $[29,61,63,64,67-$ 73, 77-80, 110] (Fig. 1).

\section{Data extraction}

\section{Demographics}

A total of 1424 patients (776 males and 648 females) were considered, with 732 patients in the accelerated group and 692 in the conservative group. The average age (mean \pm standard deviation) was $56.1 \pm 8.7$ and $56.6 \pm 9$ in the accelerated and conservative group. The mean follow-up was 12.5 months ranging from 2 [77] to 24 [63, 72, 78, 79] months. Study characteristics are reported in Table 1.

\section{Clinical outcomes, range of motions and retear rate}

As shown in Table 2, several clinical scores and ROMs have been measured in the included studies. Regarding clinical outcomes, the scoring systems usually used were the CMS measured in 9 out 16 studies $[61,63,64,68,69,72,73,77,110]$, then SST score in 5 out 16 studies $[63,64,68,70,110]$, and ASES score in 5 out 16 studies [63, 64, 68, 70, 77]. ROMs were reported in 14 out 16 studies $[29,61,63,64,67$, $68,70,72,73,77-80,110]$.

The outcome measures have been extracted by time-point follow-up from all the studies (Tables 3, 4, 5, 6, 7 and 8).

\section{Quality assessment results}

The reliability between pairs was a substantial agreement with a kappa of 0.75 ( $p$-value $<0.001$; CI $95 \%$ : 


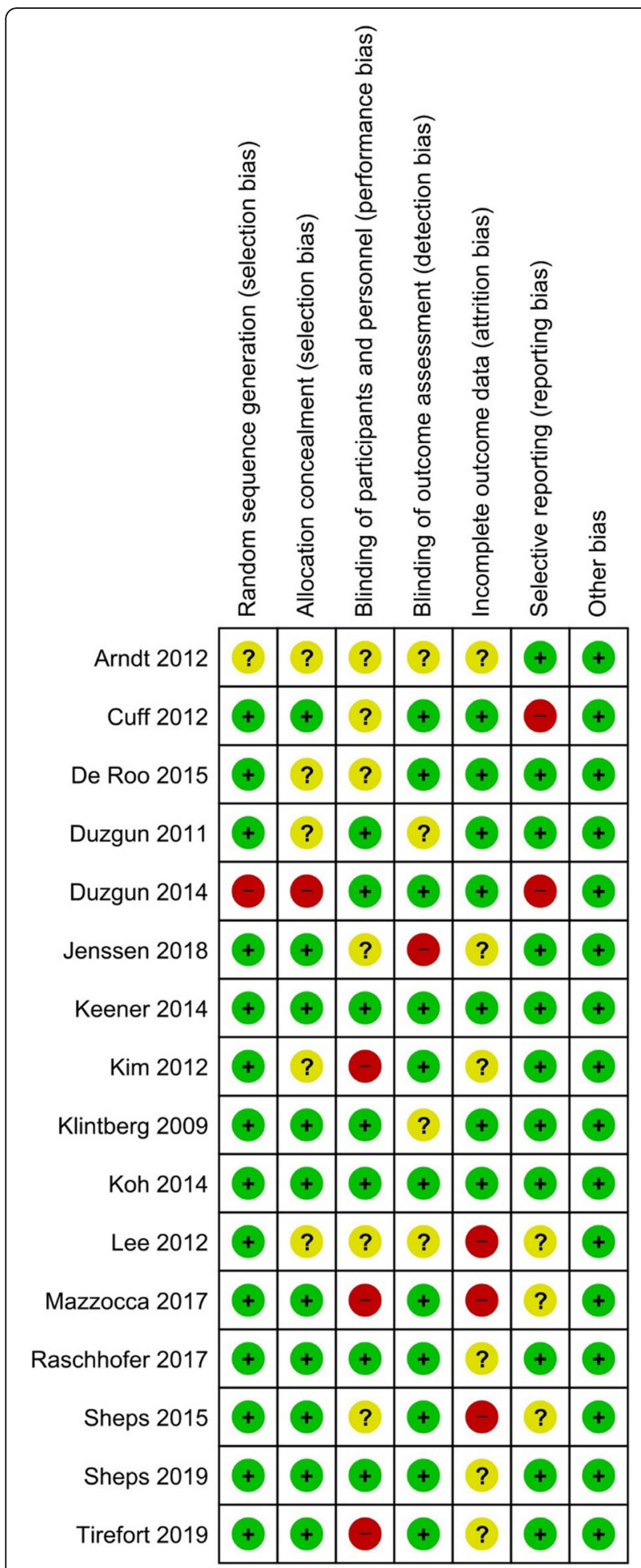

Fig. 2 Risk of bias summary: review authors' judgements about each risk of bias item for each included study

0.62-0.87). We judged ten out of 16 studies as having a low risk of bias for selection bias because they reported using an appropriate method to generate the allocation schedule [63, 68-70, 72, 73, 77-80]. Only one study was considered as high risk since a quasirandom sequence generation was used [62]. The allocation concealment was unclear in the remaining five studies [61, 64, 67, 71, 110].

Due to the lack of blinding of the patient and personnel, three studies were judged as high risk for performance bias $[64,68,80]$; whereas the blinding of participants and personnel was not described in six studies $[61,67,70,73,78,110]$. Regarding the detection bias domain, we judged eleven studies as having a low risk of bias because of the blinding of outcome assessors [29, $63,64,68-70,77-80,110]$.

We judged three trials as having a high risk of bias for incomplete outcomes data since they reported more than $20 \%$ loss to follow-up $[67,68]$ and unbalanced loss among the groups [78]. Two studies were judged as having a high risk of bias because outcomes were reported incompletely, so they cannot be entered in a meta-analysis [29, 70]. Other potential bias was not identified.

Please see the risk of bias summary presented in the figures (Figs. 2 and 3).

We provided an assessment of GRADE quality of evidence. The quality of the evidence of the included studies was found to be high.

\section{Meta-analysis}

Each study evaluated the shoulder function through several outcomes (e.g., American Society of Shoulder and Elbow Surgeon, Simple Shoulder Test, Western Ontario Rotator Cuff Index, Disabilities of the Arm, Shoulder, and Hand Questionnaire, Range of Motion). Meta-analysis was performed to investigate potential differences between conservative and accelerated rehabilitation protocol after $\mathrm{RC}$ repair in retear rate, CMS and ROMs (forward elevation, external rotation, and abduction) at 3, 6, 12, and 24 months of follow-up (Table 9).

To determine the clinical reliability of findings arising from the quantitative analysis, thresholds for MCID were established a priori. In agreement with the literature, MCID for CMS was 6.3 points, whereas MCID for abduction, forward flexion, and external rotation were $7^{\circ}$, $12^{\circ}$, and $3^{\circ}$, respectively $[111,112]$.

\section{Retear rate}

Surgical failures were reported in 10 out 16 studies $[29,61,63,64,67,68,70,77,79,110]$. Re-tears occurred in 44 of $464(9.5 \%)$ patients in the conservative group, whereas in 57 of $501(11.4 \%)$ patients in accelerated one (Table 3). The meta-analysis showed no statistically significant differences in terms of 


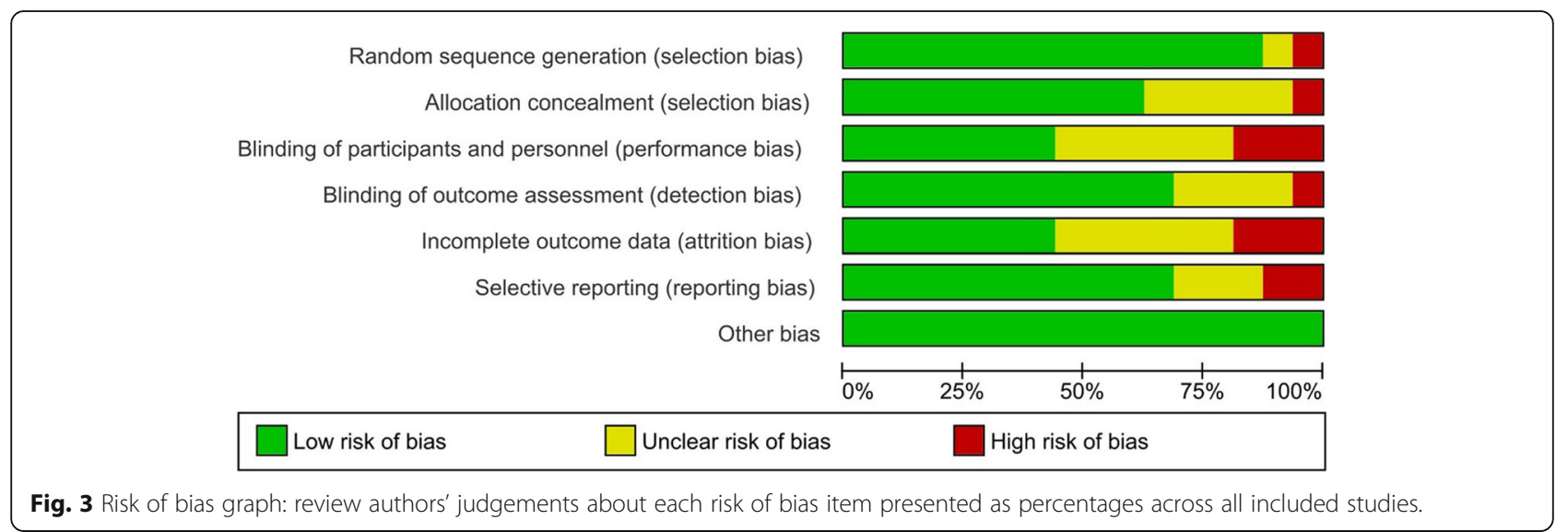

retear rate between patients who followed the accelerated rehabilitation protocol and those treated conservatively $(P=0.29)$ (Fig. 4) [113].

\section{CMS and ROMs at 3-month follow-up}

No statistically significant difference in CMS was shown at the 3 -month follow-up $(P=0.20)$. Furthermore, the quantitative analysis showed no difference between the groups in terms of abduction and forward flexion. On the other hand, the accelerated group provided statistically and clinically better results in terms of external rotation than the conservative group $(P<0.05)$ (Figs. 5, 6 and 7). The 3-month follow-up data extracted from all the included studies are shown in Table 4 [113].

\section{CMS and ROMs at 6-month follow-up}

No statistically significant difference in CMS was shown at the 6-month follow-up $(P=0.34)$. Significant statistical differences were found in favour of the accelerated group in terms of forward elevation, external rotation and abduction at 6-month follow-up $(P<0.05)$ (Figs. 8, 9 and 10). However, based on the a priori established MCID threshold, clinical reliability was only found for external rotation movement. The 6-month follow-up data extracted from all the included studies are shown in Table 5 [113].

Table 9 Summary of meta-analysis data

\begin{tabular}{|c|c|c|c|c|c|}
\hline Variables & $\begin{array}{l}\text { Follow-up } \\
\text { (months) }\end{array}$ & $\begin{array}{l}\text { No. patients accelerated } \\
\text { group }\end{array}$ & $\begin{array}{l}\text { No. patients conservative } \\
\text { group }\end{array}$ & $\begin{array}{l}\text { Mean differences (95\% } \\
\mathrm{Cl} \text { ) }\end{array}$ & $p$-value \\
\hline \multirow[t]{4}{*}{ Forward elevation } & 3 & 546 & 520 & $4.71(-0.82,10.24)$ & 0.10 \\
\hline & 6 & 553 & 527 & $3.77(2.65,4.89)$ & $\begin{array}{l}< \\
0.001^{*}\end{array}$ \\
\hline & 12 & 494 & 466 & $6.43(0.27,12.60)$ & $0.04^{*}$ \\
\hline & 24 & 261 & 248 & $1.51(-1.17,4.19)$ & 0.27 \\
\hline \multirow[t]{4}{*}{ External rotation } & 3 & 346 & 325 & $5.41(1.43,9.39)$ & $0.008^{*}$ \\
\hline & 6 & 346 & 325 & $3.19(2.20,4.81)$ & $\begin{array}{l}< \\
0.001 *\end{array}$ \\
\hline & 12 & 287 & 264 & $1.31(-2.55,5.17)$ & 0.51 \\
\hline & 24 & - & - & - & - \\
\hline \multirow[t]{4}{*}{ Abduction } & 3 & 309 & 308 & $6.61(-1.55,14.77)$ & 0.11 \\
\hline & 6 & 377 & 368 & $1.69(-0.02,3.41)$ & $0.05^{*}$ \\
\hline & 12 & 358 & 347 & $-1.48(-4.22,1.26)$ & 0.29 \\
\hline & 24 & 261 & 248 & $0.31(-2.48,3.11)$ & 0.83 \\
\hline \multirow{4}{*}{$\begin{array}{l}\text { Constant-Murley } \\
\text { Score }\end{array}$} & 3 & 161 & 149 & $3.71(-1.93,9.36)$ & 0.20 \\
\hline & 6 & 229 & 209 & $2.79(-2.91,8.49)$ & 0.34 \\
\hline & 12 & 215 & 194 & $-0.07(-0.77,0.63)$ & 0.84 \\
\hline & 24 & 68 & 60 & $0.02(-3.76,3.80)$ & 0.99 \\
\hline
\end{tabular}




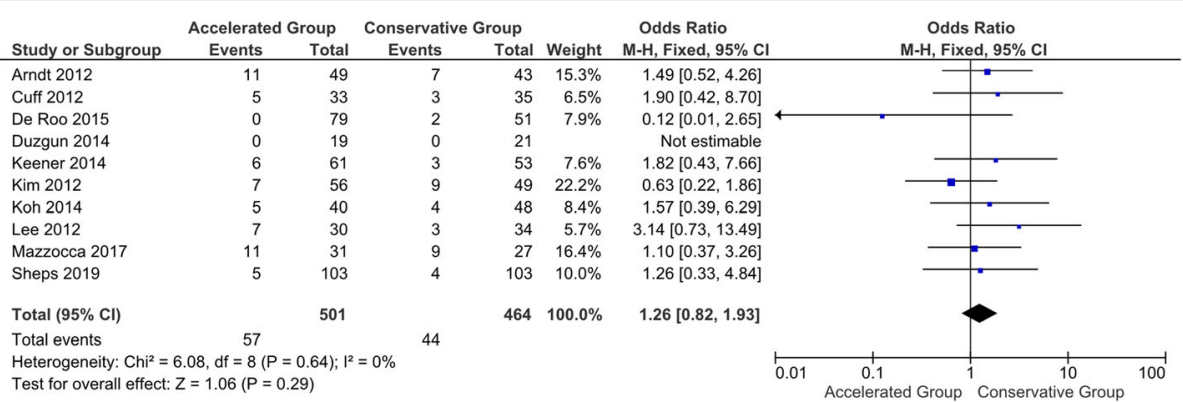

Fig. 4 Forest plot of retear. No statistically significant differences were found in retear rate between patients who followed the accelerated rehabilitation protocol and those treated conservatively $\left(P=0.29 ; 1^{2}=0 \%\right)$.

\section{CMS and ROMs at 12-month follow-up}

No statistically significant difference in CMS was shown at the 12-month follow-up $(P=0.84)$. One year after the intervention, the superiority of the accelerated group was found only in terms of forward elevation $(P<0.05)$ (Fig. 11). However, based on the a priori established MCID threshold, clinical reliability can not be sustained. All 12-month follow-up data are described in Table 6 [113].

\section{CMS and ROMs at 24-month of follow-up}

Only 4 out of 16 studies conducted a 24-month follow-up (Table 7). The meta-analysis showed no significant differences between the two group in terms of CMS or ROMs. The funnel plots of all comparisons are shown in Fig. 12.

\section{Discussion}

Pros and cons of accelerated and conservative rehabilitation protocols after arthroscopic RC repair have been reported [4, 53, 92-98]. Based on the available evidence, the American Academy of Orthopaedic Surgeons (AAOS) could not draw definitive conclusions for the best rehabilitation protocol [114]. The purpose of the present systematic review was to identify a potential superiority of either form of rehabilitation in clinical outcomes and ROMs at 3, 6, 12, and 24-month follow-up.
The meta-analysis highlighted clinically and statistically significant differences in favour of accelerated rehabilitation in terms of external rotation at three months of follow-up. The conservative group demonstrated worse results in terms of abduction, forward elevation and external rotation at six months follow-up than the accelerated group. However, regarding clinical interpretability, thresholds for MCID were achieved only for external rotation. The quantitative analysis showed a mean difference for forward elevation and abduction of 3.77 and 1, 61, respectively. Despite their statistical significance, these results may lack clinical relevance because they do not exceed MCID. Besides, forward elevation at 12month follow-up was statistically but not clinically superior in the accelerated group than in the conservative one. Only four studies recorded two-year follow-up revealing comparable efficacy among accelerated and conservative rehabilitation protocols $[63,72,78,79]$.

During the first postoperative weeks, the tendon healing process occurs through the first inflammatory phase (first week), followed by the second stage of cell proliferation (second and third week) and, finally, the maturation and restoration to original condition period (up to 6 months) $[115,116]$. Shoulder immobilization has always been considered a critical step in the treatment protocol to balance the preservation of repair integrity and restoration of function in patients undergoing $\mathrm{RC}$

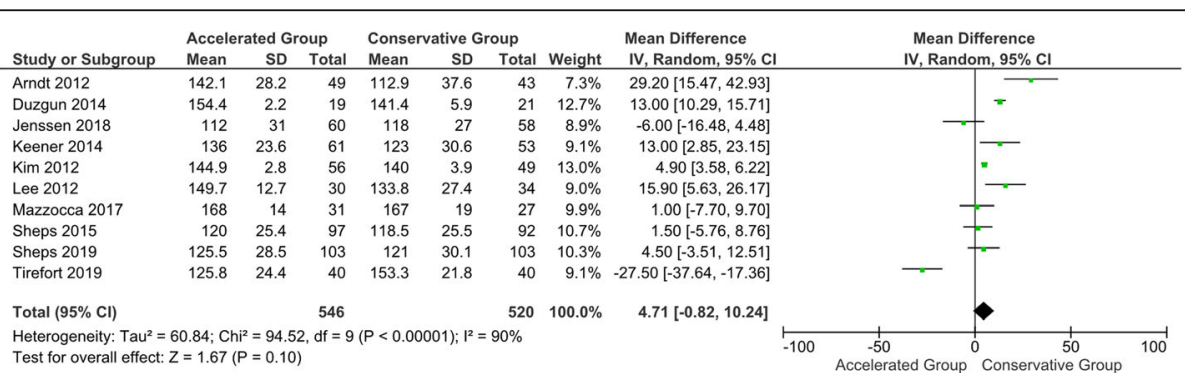

Fig. 5 Forest plot of forward elevation at 3-month follow-up. No statistically significant differences were found in forward elevation between patients who followed the accelerated rehabilitation protocol and those treated conservatively $\left(P=0.1 ;\left.\right|^{2}=90 \%\right)$ 


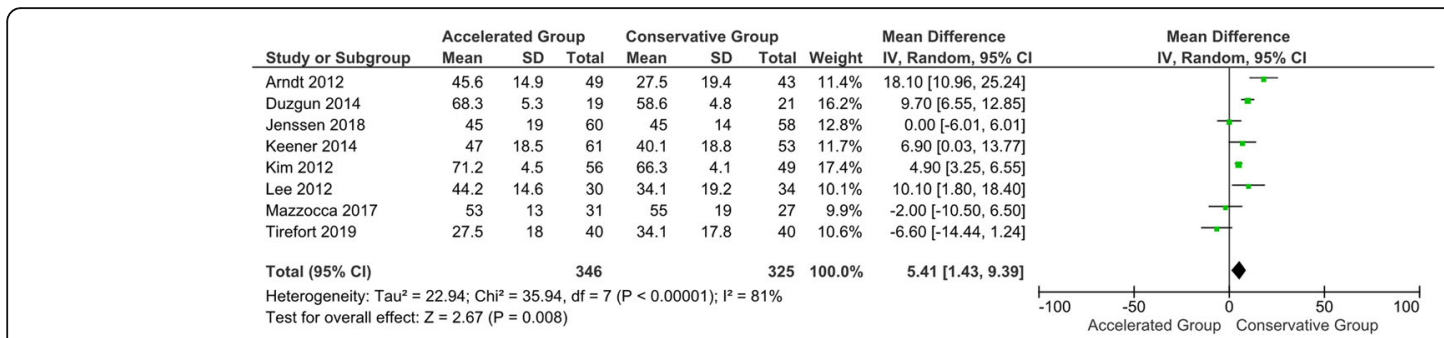

Fig. 6 Forest plot of external rotation at 3-month follow-up. The accelerated group provide better results in external rotation than conservative group $\left(P<0.05 ; 1^{2}=81 \%\right)$

repair $[26,74,117]$. However, histopathological changes responsible for joint stiffness arising from prolonged immobilization have been demonstrated $[115,118,119]$. To avoid the negative effect of immobilization and to support a quick reintegration into daily activities, an accelerated protocol enabling movements before six weeks can be recommended [42, 64, 71]. However, the first clinical results revealed that patients following an accelerated rehabilitation protocol experienced an increased number of retear events [120-122]. Among the ten included studies that reported retear events, only Arndt et al., Cuff et al., and Lee et al. demonstrated a slight and not statistically significant improvement in the tendon healing process with immobilization [61, 67, 70]. However, based on both our results and those of the previous meta-analyses, there are no statistically significant differences in the retear rate among the accelerated and conservative groups $[53,65,66,93]$.

This systematic review included 14 studies providing different ROMs results between conservative and accelerated rehabilitation groups after RC repair. The majority of available studies have shown comparable results among the groups $[29,63,64,70,73,77,79,110]$. Interestingly, when data has stratified for time-points followup, Arndt et al., Lee et al., and Tirefort et al. reported improvements in shoulder function at 6-month followup in patients who performed accelerated rehabilitation $[61,67,80]$. Moreover, according to the findings of this meta-analysis, the accelerated group's superiority in terms of external rotation was demonstrated both at three and 6-month follow-up. Previous studies have shown that the tendon healing process requires the first six postoperative months and that several variables which may occur during this period can influence the outcome. [75, 76, 115, 116, 123]. In the short-term, our results in terms of ROMs were demonstrated by a previous meta-analysis [96].

Interestingly, when the comparison was adjusted for the tear size (i.e. large lesion), Chang et al. observed a statistically significant trend towards an increased number of retear events in the accelerated group [96]. Therefore, as tear size, several other factors may influence the early postoperative rehabilitation outcomes [124]. Indeed, minor injuries are related to all ROMs' progress, large lesions with all ROMs except flexion [65, 124]. However, the potential role of the tear size is still debate. As regards small and medium lesions (< $5 \mathrm{~cm}$ ), the arm immobilization for six weeks following arthroscopic RC treatment seems to improve tendon healing [125]. However, prolonged immobilization did not provide significant shoulder function advantages [67, 77, 126, 127]. Even though the accelerated rehabilitation protocol seems to improve ROMs, a decrease in tendon healing has been reported in tears sized $3-5 \mathrm{~cm}$ [128]. Therefore, the impact of tear size in terms of clinical outcomes requires further investigation.

The present meta-analysis showed a reduction of statistically significant results in the long term. A better forward flexion at 12-month follow-up in favour of accelerated rehabilitation protocol was detected. However, the clinical reliability of this finding is weak. Among the ten studies that made the comparison one year after

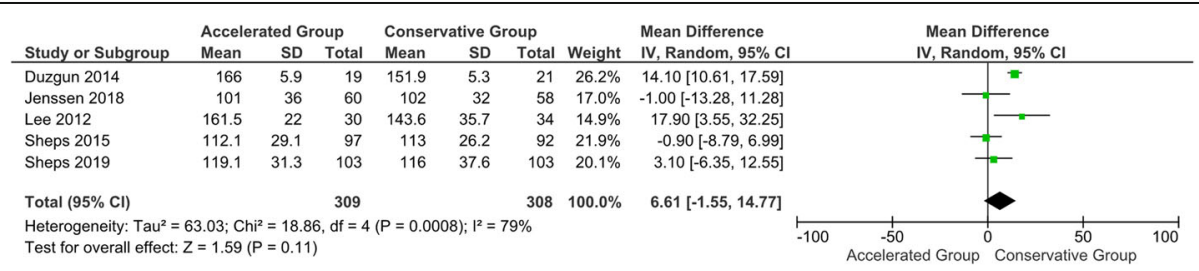

Fig. 7 Forest plot of abduction at 3-month follow-up. No statistically significant differences were found in abduction between patients who followed the accelerated rehabilitation protocol and those treated conservatively $\left(P=0.11 ; 1^{2}=79 \%\right)$ 


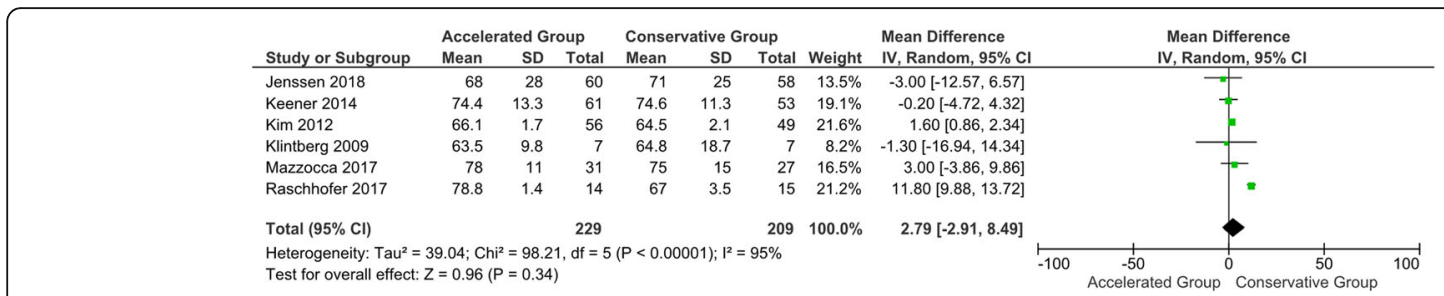

Fig. 8 Forest plot of CMS at 6-month follow-up. No statistically significant differences were found in Constant-Murley score between patients who followed the accelerated rehabilitation protocol and those treated conservatively $\left(P=0.34 ;\left.\right|^{2}=95 \%\right)$

surgery, only Arndt et al. reported slightly worse results for the conservative group in terms of external rotation [61]. In contrast, the remaining studies found comparable effects on final shoulder function. Comparable findings were assessed at 24 months of follow-up between the groups suggesting that the two rehabilitation protocols could be equally safe and effective. However, this result may be influenced by the lack of studies with longterm follow-up. To date, only four high-level studies have analysed the effectiveness of the two forms of rehabilitation protocol two years after the surgery without showing statistically significant results $[63,72,78,79]$. Therefore, further long-term studies are essential to define the superiority of one of the two forms of rehabilitation protocol.

Although rigorous methods were used for this systematic review, and only level I-II studies were selected to increase the strength of the results, some limitations should be outlined. Whereas the conservative protocol applied was the same in all studies, the accelerated group performed protocols with different exercises and sessions per week (Table 10). In several studies, the first postoperative movements were performed with pendulum exercises or passive mobilization with rope, pulley, or cane. A comparison of these exercises was not possible. Besides, active mobilization was started at different time points in different studies. In the immediate postoperative period, only two studies allowed active shoulder motion [64, 68]. In the other studies, active ROMs were allowed at three week [29, 73], at four week [72, 80], at five week [110], and at six week postoperatively $[61,63,67,70]$, whereas in three studies the beginning of active ROMs was unspecified or unclear $[69,77$, 79]. Shoulder muscle strengthening was allowed after 4-6 weeks postoperatively in four studies [62, 67, 69, 72], after 8-9 weeks in two studies [64, 110], after three months in three studies [63, 70, 80], after four months in two studies [61, 77], while in the remaining three it was not specified or was unclear [68, 73, 79]. Given this wide heterogeneity, it was not possible to perform a sub-analysis between protocols. Therefore, we are unable to quantify whether these differences may have influenced our results. To decrease the sample population's heterogeneity, only level I-II studies evaluating the two forms of rehabilitation after arthroscopic RC repair were examined. However, due to the lack of information on the RC tear characteristics (e.g. tear size) in many studies, we could not conduct a subgroup analysis. Furthermore, the RC tear's chronicity or the level of muscle atrophy and fatty infiltration was not specified in most of the included articles. Moreover, those articles that provided these data different, and not comparable, classification systems were used.

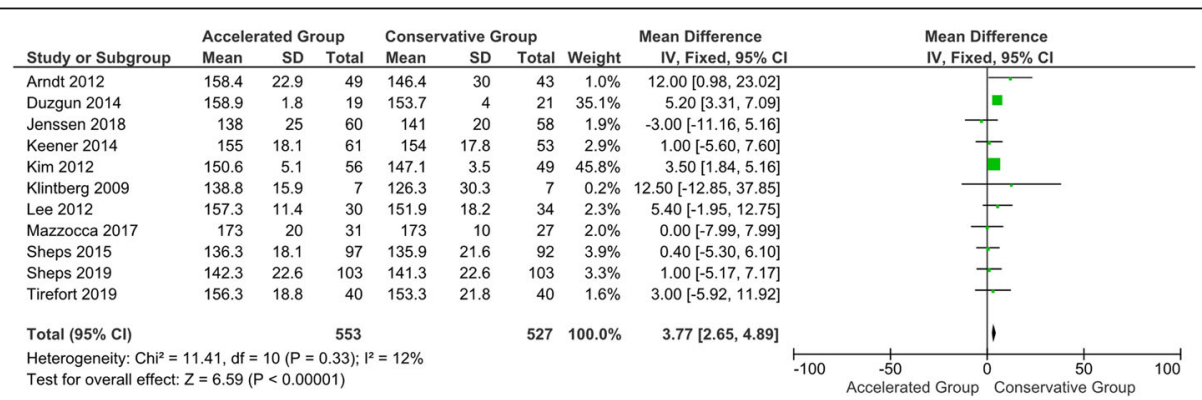

Fig. 9 Forest plot of forward elevation at 6-month follow-up. The accelerated group provide better results in forward elevation than conservative $\operatorname{group}\left(P<0.05 ;\left.\right|^{2}=12 \%\right)$ 


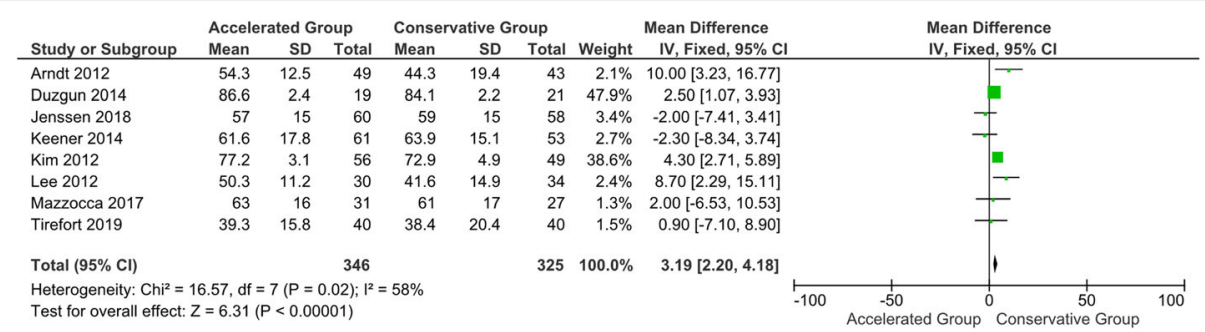

Fig. 10 Forest plot of external rotation at 6-month follow-up. The accelerated group provide better results in external rotation than conservative group $\left(P<0.05 ;\left.\right|^{2}=58 \%\right)$

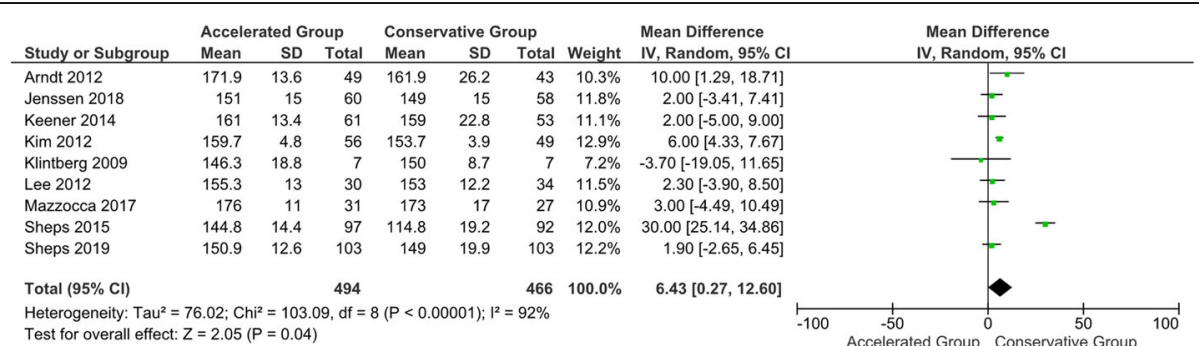

Fig. 11 Forest plot of forward elevation at 12-month follow-up. The accelerated group provide better results in forward elevation than conservative group $\left(P<0.05 ;\left.\right|^{2}=92 \%\right)$

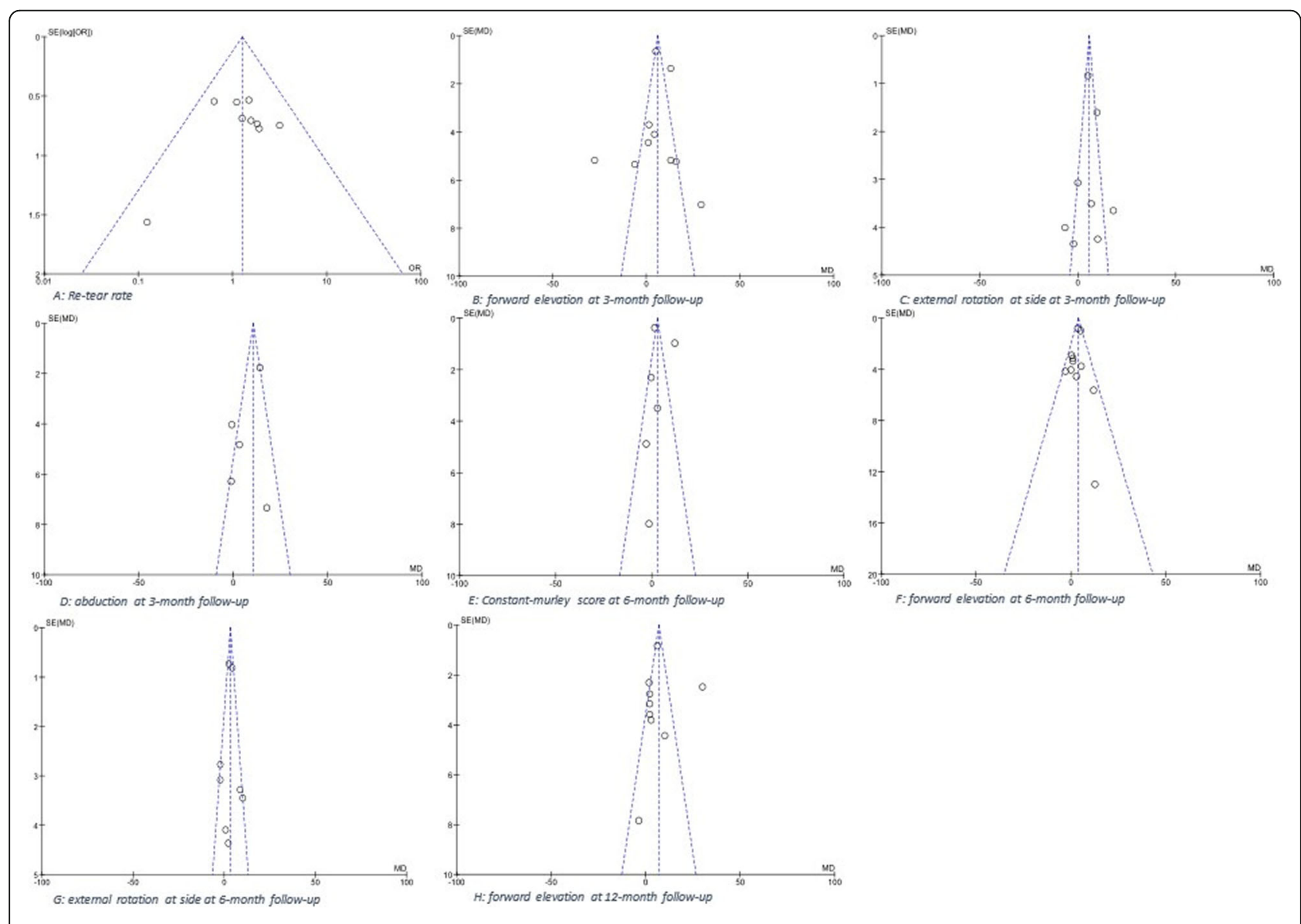

Fig. 12 Funnel plots of comparisons: conservative versus accelerated rehabilitation protocols 
Table 10 Comparison of accelerated rehabilitation protocols

\begin{tabular}{|c|c|}
\hline Authors & Accelerated rehabilitation protocol \\
\hline Arndt et al. [61] & $\begin{array}{l}\text { Shoulder passive ROM and pendulum exercise } 1 \mathrm{~d} \text { postoperatively ( } 3-5 \text { times/wk); active shoulder rehabilitation } 6 \text { wk } \\
\text { postoperatively; shoulder muscle strengthening } 4 \text { mo postoperatively }\end{array}$ \\
\hline Cuff et al. [70] & $\begin{array}{l}\text { Shoulder passive ROM and pendulum exercise } 2 \mathrm{~d} \text { postoperatively ( } 3 \text { times/ wk and } 3 \text { times/d, respectively); active ROM of } \\
\text { elbow, wrist, and hand immediately after surgery; aa shoulder exercise } 6 \text { wk postoperatively ( } 3 \text { times/wk); rotator cuff } \\
\text { strengthening } 12 \text { wk postoperatively }\end{array}$ \\
\hline $\begin{array}{l}\text { De Roo et al. } \\
{[110]}\end{array}$ & $\begin{array}{l}\text { Shoulder passive ROM and pendulum exercises } 1 \mathrm{~d} \text { postoperatively ( } 3-5 \text { times/wk). Specific capsular glenohumeral exercises } \\
\text { and progressively active ROM } 5 \text { wk postoperatively. Rotator cuff strengthening } 8 \text { wk postoperatively }\end{array}$ \\
\hline $\begin{array}{l}\text { Düzgün et al. }[29, \\
71]\end{array}$ & $\begin{array}{l}\text { Shoulder passive ROM } 1 \text { wk postoperatively ( } 1 \text { time/d at clinic and } 2 \text { times/d at home); active ROM of elbow, hand, and nec } \\
\text { wk postoperatively; active shoulder rehabilitation } 3 \text { wk postoperatively; rotator cuff strengthening } 4 \text { wk postoperatively }\end{array}$ \\
\hline Jenssen et al. [73] & Shoulder passive ROM and pendulum exercises $1 \mathrm{~d}$ postoperatively; shoulder active ROM 3 wk postoperatively \\
\hline Keener et al. [63] & $\begin{array}{l}\text { Pendulum exercise and active ROM of elbow, wrist, and hand immediately after surgery; shoulder passive ROM } 1 \text { wk } \\
\text { postoperatively; shoulder active ROM } 6 \text { wk postoperatively; shoulder muscle strengthening } 3 \text { mo postoperatively; full activit } \\
\text { mo postoperatively }\end{array}$ \\
\hline Kim et al. [64] & $\begin{array}{l}\text { Shoulder passive ROM } 2 \text { d postoperatively; shrugging shoulder exercise and active ROM of elbow, forearm, wrist, and hand } \\
\text { immediately after surgery; aa shoulder exercise after weaning of immobilizer; shoulder muscle strengthening } 9 \text { wk } \\
\text { postoperatively; sport activities } 6 \text { mo postoperatively }\end{array}$ \\
\hline $\begin{array}{l}\text { Klintberg et al. } \\
{[72]}\end{array}$ & low-level active ROM 2 d postoperatively; active assisted ROM 4 weeks, aquatic training, loading at 6 weeks \\
\hline Koh et al. [77] & $\begin{array}{l}4 \text { weeks of immobilization without passive ROM; shoulder passive ROM with rope, pulley, and cane } 4 \text { wk postoperatively; } \\
\text { shoulder muscle strengthening } 11 \text { wk postoperatively }\end{array}$ \\
\hline Lee et al. [67] & $\begin{array}{l}\text { Shoulder passive ROM exercise up to tolerable angle } 1 \mathrm{~d} \text { postoperatively ( } 2 \text { times/d by physical therapist and } 3 \text { times/d by } \\
\text { patient, respectively); shoulder active ROM } 6 \text { wk postoperatively; shoulder muscle strengthening } 6 \text { wk postoperatively; } \\
\text { recreational activities with heavy demands } 6 \text { mo postoperatively }\end{array}$ \\
\hline
\end{tabular}

Mazzocca et al. Shoulder active ROM with a cane 2-3 d postoperative

[68]

Raschhofer et al. Shoulder isometric activation (i.e. the dynamic relocation test) 2-6wk postoperatively; shoulder muscle strengthening 6 wk [69] postoperatively

Sheps et al. [78, Shoulder passive ROM and pain-free activities only, with the exception of resisted activities, at discharge 79]

Tirefort et al. [80] Shoulder passive ROM during the first 4 postoperative weeks. Active ROM 4 wk postoperatively; demanding activities and light sports were authorized after 2 months, and a strengthening program was permitted only after 3 postoperative months.

ROM Range of motion, $d$ day, wk week, mo month

\section{Conclusions}

No statistically significant differences in the retear rate among the accelerated and conservative group have been demonstrated. On the other hand, statistically and clinically significant differences were found in terms of external rotation at 3 and 6 months of follow-up in favour of the accelerated group. However, no differences between the two groups were detected at 24 months follow-up. Further long-term studies are warranted to define the superiority between the accelerated and conservative rehabilitation protocol.

\footnotetext{
Abbreviations

RC: Rotator cuff; ROM: Range of motion; MeSH: Medical Subject Headings; CMS: Constant-Murley score; SST: Simple Shoulder Test; ASES: American Shoulder and elbow surgeons; VAS: Visual analog scale; AAOS: American Academy of Orthopaedic Surgeons; MCID: Minimal clinically important difference
}

\section{Authors' contributions}

Conceptualization, U.G.L. and V.D.; methodology, L.R.A., software, A.B. validation, A.B., F.M. and V.C.; formal analysis, A.C and E.S.; investigation, JDS.; resources, V.C.; data curation, L.R.A; writing —original draft preparation, L.R.A, AN, JDS.; writing - review and editing, V.C. and F.M., A.B., visualization, A.B.; supervision, V.D.; project administration, U.G.L. Each author has approved the current version and has agreed both to be personally responsible for his or her own contributions and for issues related to the accuracy or integrity of any part of the work. The author(s) read and approved the final manuscript.

\section{Funding}

This research received no external funding.

\section{Availability of data and materials}

The datasets used and/or analysed during the current study available from the corresponding author on reasonable request.

\section{Declarations}

Ethics approval and consent to participate Not applicable. 


\section{Competing interests}

U.G.L. is a member of the Editorial Board of B.M.C. Musculoskeletal Disorders. The remaining authors declare that they have no conflict of interest.

\section{Author details Boston, MA, USA. \\ Received: 17 August 2020 Accepted: 25 May 2021 \\ Published online: 24 July 2021}

'Department of Orthopaedic and Trauma Surgery, Campus Bio-Medico University, Via Alvaro del Portillo, 200, Rome, Trigoria 00128, Italy. ${ }^{2}$ Research Unit of Measurements and Biomedical Instrumentation, Campus Bio-Medico University, Via Alvaro del Portillo, 200, Rome, Trigoria 00128, Italy. ${ }^{3}$ Center for Advanced Orthopaedic Studies, Carl J. Shapiro Department of Orthopaedic Surgery, Beth Israel Deaconess Medical Center, Harvard Medical School,

\section{References}

1. Longo UG, Berton A, Papapietro N, Maffulli N, Denaro V. Epidemiology, genetics and biological factors of rotator cuff tears. Med Sport Sci. 2012;57: $1-9$.

2. Salvatore G, Longo UG, Candela V, Berton A, Migliorini F, Petrillo S, Ambrogioni LR, Denaro V. Epidemiology of rotator cuff surgery in Italy: regional variation in access to health care. Results from a 14-year nationwide registry. Musculoskelet Surg. 2020;104(3):329-35. https://doi. org/10.1007/s12306-019-00625-y.

3. Longo UG, Salvatore G, Rizzello G, Berton A, Ciuffreda M, Candela V, Denaro $\mathrm{V}$. The burden of rotator cuff surgery in Italy: a nationwide registry study. Arch Orthop Trauma Surg. 2017:137(2):217-24.

4. Dickinson RN, Kuhn JE, Bergner JL, Rizzone KH. A systematic review of costeffective treatment of postoperative rotator cuff repairs. J Shoulder Elbow Surg. 2017;26(5):915-22.

5. Del Buono A, Oliva F, Longo UG, Rodeo SA, Orchard J, Denaro V, Maffulli N. Metalloproteases and rotator cuff disease. J Shoulder Elbow Surg. 2012; 21(2):200-8

6. Longo UG, Berton A, Khan WS, Maffulli N, Denaro V. Histopathology of rotator cuff tears. Sports Med Arthrosc. 2011;19(3):227-36.

7. Longo UG, Forriol F, Campi S, Maffulli N, Denaro V. Animal models for translational research on shoulder pathologies: from bench to bedside. Sports Med Arthrosc. 2011;19(3):184-93.

8. Maffulli N, Longo UG, Berton A, Loppini M, Denaro V. Biological factors in the pathogenesis of rotator cuff tears. Sports Med Arthrosc. 2011;19(3):194201.

9. Denaro V, Ruzzini L, Longo UG, Franceschi F, De Paola B, Cittadini A, Maffulli $\mathrm{N}$, Sgambato A. Effect of dihydrotestosterone on cultured human tenocytes from intact supraspinatus tendon. Knee Surg Sports Traumatol Arthrosc. 2010;18(7):971-6.

10. Maffulli N, Margiotti K, Longo UG, Loppini M, Fazio VM, Denaro V. The genetics of sports injuries and athletic performance. Muscles Ligaments Tendons J. 2013;3(3):173-89.

11. Longo UG, Franceschi F, Ruzzini L, Spiezia F, Maffulli N, Denaro V. Higher fasting plasma glucose levels within the normoglycaemic range and rotator cuff tears. Br J Sports Med. 2009:43(4):284-7.

12. Carnevale A, Longo UG, Schena E, Massaroni C, Lo Presti D, Berton A, Candela V, Denaro V. Wearable systems for shoulder kinematics assessment: a systematic review. BMC Musculoskelet Disord. 2019;20(1):546.

13. Longo UG, Candela V, Berton A, Salvatore G, Guarnieri A, DeAngelis J, Nazarian A, Denaro V. Genetic basis of rotator cuff injury: a systematic review. BMC Med Genet. 2019;20(1):149.

14. Longo UG, Margiotti K, Petrillo S, Rizzello G, Fusilli C, Maffulli N, De Luca A, Denaro V. Genetics of rotator cuff tears: no association of col5al gene in a case-control study. BMC Med Genet. 2018;19(1):217.

15. Franceschi F, Longo UG, Ruzzini L, Rizzello G, Maffulli N, Denaro V. Soft tissue tenodesis of the long head of the biceps tendon associated to the Roman Bridge repair. BMC Musculoskelet Disord. 2008;9:78.

16. Longo UG, Franceschi F, Spiezia F, Marinozzi A, Maffulli N, Denaro V. The low-profile Roman bridge technique for knotless double-row repair of the rotator cuff. Arch Orthop Trauma Surg. 2011;131(3):357-61.

17. Franceschi F, Longo UG, Ruzzini L, Rizzello G, Maffulli N, Denaro V. The Roman Bridge: a "double pulley - suture bridges" technique for rotator cuff repair. BMC Musculoskelet Disord. 2007;8:123.
18. Khan WS, Adesida AB, Tew SR, Longo UG, Hardingham TE. Fat pad-derived mesenchymal stem cells as a potential source for cell-based adipose tissue repair strategies. Cell Prolif. 2012;45(2):111-20.

19. Lommi M, Matarese M, Alvaro R, Piredda M, De Marinis MG. The experiences of self-care in community-dwelling older people: a meta-synthesis. Int J Nurs Stud. 2015:52(12):1854-67.

20. Piredda M, Vellone E, Piras G, Fida R, Latour JM, Matarese M, Alvaro R, De Marinis MG. Psychometric evaluation of the Newcastle Satisfaction with Nursing Scales. J Nurs Care Qual. 2015;30(1):84-92.

21. Belletti M, Mallia L, Lucidi F, Reichmann S, Mastroianni C, De Marinis MG, Casale G. Complementary therapy and support services for formal and informal caregivers in Italian palliative care hospices: an exploratory and descriptive study. Support Care Cancer. 2011;19(12):1939-47.

22. Pennacchini M, Bertolaso M, Elvira MM, De Marinis MG. A brief history of the quality of Life: its use in medicine and in philosophy. Clin Ter. 2011;162(3): e99-103.

23. Longo UG, Facchinetti G, Marchetti A, Candela V, Risi Ambrogioni L, Faldetta A, De Marinis MG, Denaro V. Sleep disturbance and rotator cuff tears: a systematic review. Medicina (Kaunas). 2019;55(8):453. https://doi.org/10.33 90/medicina55080453.

24. Longo UG, Rizzello G, Petrillo S, Loppini M, Maffulli N, Denaro V. Conservative rehabilitation provides superior clinical results compared to early aggressive rehabilitation for rotator cuff repair: a retrospective comparative study. Medicina (Kaunas). 2019;55(8):402. https://doi.org/10.33 90/medicina55080402

25. Longo UG, Ciuffreda M, Locher J, Buchmann S, Maffulli N, Denaro V. The effectiveness of conservative and surgical treatment for shoulder stiffness: a systematic review of current literature. Br Med Bull. 2018;127(1):111-43.

26. Osborne JD, Gowda AL, Wiater B, Wiater JM. Rotator cuff rehabilitation: current theories and practice. Phys Sportsmed. 2016:44(1):85-92.

27. Longo UG, Franceschi F, Berton A, Maffulli N, Droena V. Conservative treatment and rotator cuff tear progression. Med Sport Sci. 2012;57:90-9.

28. Longo UG, Petrillo S, Candela V, Rizzello G, Loppini M, Maffulli N, Denaro V. Arthroscopic rotator cuff repair with and without subacromial decompression is safe and effective: a clinical study. BMC Musculoskelet Disord. 2020:21(1):24

29. Düzgün İ, Baltacı G, Turgut E, Atay OA. Effects of slow and accelerated rehabilitation protocols on range of motion after arthroscopic rotator cuff repair. Acta Orthop Traumatol Turc. 2014;48(6):642-8.

30. De Carli A, Fabbri M, Lanzetti RM, Ciompi A, Gaj E, Beccarini G, Vetrano M, Ferretti A. Functional treatment in rotator cuff tears: is it safe and effective? A retrospective comparison with surgical treatment. Muscles Ligaments Tendons J. 2017;7(1):40-5.

31. Nazari G, MacDermid JC, Bryant D, Athwal GS. The effectiveness of surgical vs conservative interventions on pain and function in patients with shoulder impingement syndrome. A systematic review and meta-analysis. PLoS One. 2019;14(5):e0216961.

32. Carr A, Cooper C, Campbell MK, Rees J, Moser J, Beard DJ, Fitzpatrick R, Gray A, Dawson J, Murphy J, et al. Effectiveness of open and arthroscopic rotator cuff repair (UKUFF): a randomised controlled trial. Bone Joint J. 2017;99-B(1):107-15.

33. Cho $\mathrm{CH}$, Song KS, Jung GH, Lee YK, Shin HK. Early postoperative outcomes between arthroscopic and mini-open repair for rotator cuff tears. Orthopedics. 2012;35(9):e1347-52.

34. Liu J, Fan L, Zhu Y, Yu H, Xu T, Li G. Comparison of clinical outcomes in allarthroscopic versus mini-open repair of rotator cuff tears: a randomized clinical trial. Medicine. 2017;96(11):e6322.

35. van der Zwaal P, Thomassen BJ, Nieuwenhuijse MJ, Lindenburg R, Swen JW, van Arkel ER. Clinical outcome in all-arthroscopic versus mini-open rotator cuff repair in small to medium-sized tears: a randomized controlled trial in 100 patients with 1-year follow-up. Arthroscopy. 2013;29(2):266-73.

36. Abrams GD, Gupta AK, Hussey KE, Tetteh ES, Karas V, Bach BR, Cole BJ, Romeo AA, Verma NN. Arthroscopic repair of full-thickness rotator cuff tears with and without acromioplasty: randomized prospective trial with 2-year follow-up. Am J Sports Med. 2014;42(6):1296-303.

37. Dezaly C, Sirveaux F, Philippe R, Wein-Remy F, Sedaghatian J, Roche O, Molé D. Arthroscopic treatment of rotator cuff tear in the over-60s: repair is preferable to isolated acromioplasty-tenotomy in the short term. Orthop Traumatol Surg Res. 2011;97(6 Suppl):125-30.

38. Jacquot A, Dezaly C, Goetzmann T, Roche O, Sirveaux F, Molé D. French Society for Shoulder EbS: Is rotator cuff repair appropriate in patients older than 60 years of age? prospective, randomised trial in 103 patients with a 
mean four-year follow-up. Orthop Traumatol Surg Res. 2014;100(6 Suppl): 333-8.

39. MacDonald P, McRae S, Leiter J, Mascarenhas R, Lapner P. Arthroscopic rotator cuff repair with and without acromioplasty in the treatment of fullthickness rotator cuff tears: a multicenter, randomized controlled trial. J Bone Joint Surg Am. 2011:93(21):1953-60.

40. Mohtadi NG, Hollinshead RM, Sasyniuk TM, Fletcher JA, Chan DS, Li FX. A randomized clinical trial comparing open to arthroscopic acromioplasty with mini-open rotator cuff repair for full-thickness rotator cuff tears: disease-specific quality of life outcome at an average 2-year follow-up. Am 」 Sports Med. 2008;36(6):1043-51.

41. Shin SJ, Oh JH, Chung SW, Song MH. The efficacy of acromioplasty in the arthroscopic repair of small- to medium-sized rotator cuff tears without acromial spur: prospective comparative study. Arthroscopy. 2012;28(5):62835.

42. Conti M, Garofalo R, Delle Rose G, Massazza G, Vinci E, Randelli M, Castagna A. Post-operative rehabilitation after surgical repair of the rotator cuff. Chir Organi Mov. 2009;93(Suppl 1):55-63.

43. Huberty DP, Schoolfield JD, Brady PC, Vadala AP, Arrigoni P, Burkhart SS. Incidence and treatment of postoperative stiffness following arthroscopic rotator cuff repair. Arthroscopy. 2009:25(8):880-90.

44. Longo UG, Petrillo S, Loppini M, Candela V, Rizzello G, Maffulli N, Denaro V. Metallic versus biodegradable suture anchors for rotator cuff repair: a case control study. BMC Musculoskelet Disord. 2019;20(1):477.

45. Nazari G, MacDermid JC, Bryant D, Dewan N, Athwal GS. Effects of arthroscopic vs. mini-open rotator cuff repair on function, pain \& range of motion. A systematic review and meta-analysis. PLoS One. 2019;14(10): e0222953.

46. Moosmayer S, Lund G, Seljom U, Svege I, Hennig T, Tariq R, Smith HJ. Comparison between surgery and physiotherapy in the treatment of small and medium-sized tears of the rotator cuff: a randomised controlled study of 103 patients with one-year follow-up. J Bone Joint Surg Br. 2010;92(1):83-91.

47. Moosmayer S, Lund G, Seljom US, Haldorsen B, Svege IC, Hennig T, Pripp $\mathrm{AH}$, Smith HJ. At a 10-year follow-up, tendon repair is superior to physiotherapy in the treatment of small and medium-sized rotator cuff tears. J Bone Joint Surg Am. 2019;101(12):1050-60.

48. Kukkonen J, Joukainen A, Lehtinen J, Mattila KT, Tuominen EK, Kauko T, Äärimaa V. Treatment of nontraumatic rotator cuff tears: a randomized controlled trial with two years of clinical and imaging follow-up. J Bone Joint Surg Am. 2015;97(21):1729-37.

49. Lambers Heerspink FO, van Raay JJ, Koorevaar RC, van Eerden PJ, Westerbeek RE, van 't Riet E, van den Akker-Scheek I, Diercks RL. Comparing surgical repair with conservative treatment for degenerative rotator cuff tears: a randomized controlled trial. J Shoulder Elbow Surg. 2015;24(8):127481.

50. Kukkonen J, Joukainen A, Lehtinen J, Mattila KT, Tuominen EK, Kauko T, Aärimaa V. Treatment of non-traumatic rotator cuff tears: A randomised controlled trial with one-year clinical results. Bone Joint J. 2014;96-B(1):7581.

51. Moosmayer S, Lund G, Seljom US, Haldorsen B, Svege IC, Hennig T, Pripp $\mathrm{AH}$, Smith HJ. Tendon repair compared with physiotherapy in the treatment of rotator cuff tears: a randomized controlled study in 103 cases with a fiveyear follow-up. J Bone Joint Surg Am. 2014;96(18):1504-14.

52. Longo UG, Franceschi F, Ruzzini L, Rabitti C, Morini S, Maffulli N, Denaro V. Histopathology of the supraspinatus tendon in rotator cuff tears. Am J Sports Med. 2008;36(3):533-8.

53. Mazuquin BF, Wright AC, Russell S, Monga P, Selfe J, Richards J. Effectiveness of early compared with conservative rehabilitation for patients having rotator cuff repair surgery: an overview of systematic reviews. $\mathrm{Br} J$ Sports Med. 2018:52(2):111-21.

54. Büker N, Kitiş A, Akkaya S, Akkaya N. Comparison of the results of supervised physiotherapy program and home-based exercise program in patients treated with arthroscopic-assisted mini-open rotator cuff repair. Eklem Hastalik Cerrahisi. 2011;22(3):134-9.

55. Hayes K, Ginn KA, Walton JR, Szomor ZL, Murrell GA. A randomised clinical trial evaluating the efficacy of physiotherapy after rotator cuff repair. Aust $J$ Physiother. 2004;50(2):77-83.

56. Lisinski P, Huber J, Wilkosz P, Witkowska A, Wytrazek M, Samborski W, Zagloba A. Supervised versus uncontrolled rehabilitation of patients after rotator cuff repair-clinical and neurophysiological comparative study. Int J Artif Organs. 2012;35(1):45-54.
57. Chou CT, Hu W, Wen CS, Wang SF, Lieu FK, Teng JT. Efficacy of informed versus uninformed physiotherapy on postoperative retear rates of mediumsized and large rotator cuff tears. J Shoulder Elbow Surg. 2015;24(9):141320.

58. Roddey TS, Olson SL, Gartsman GM, Hanten WP, Cook KF. A randomized controlled trial comparing 2 instructional approaches to home exercise instruction following arthroscopic full-thickness rotator cuff repair surgery. J Orthop Sports Phys Ther. 2002;32(11):548-59.

59. Longo UG, Berton A, Risi Ambrogioni L, Lo Presti D, Carnevale A, Candela V, Stelitano G, Schena E, Nazarian A, Denaro V. Cost-effectiveness of supervised versus unsupervised rehabilitation for rotator-cuff repair: systematic review and meta-analysis. Int J Environ Res Public Health 2020;17(8):2852. https:// doi.org/10.3390/ijerph17082852.

60. Longo UG, Risi Ambrogioni L, Berton A, Candela V, Carnevale A, Schena E, Gugliemelli E, Denaro V. Physical therapy and precision rehabilitation in shoulder rotator cuff disease. Int Orthop. 2020;44(5):893-903. https://doi. org/10.1007/s00264-020-04511-2.

61. Arndt J, Clavert P, Mielcarek P, Bouchaib J, Meyer N, Kempf JF, (SOFEC) FSfSE. Immediate passive motion versus immobilization after endoscopic supraspinatus tendon repair: a prospective randomized study. Orthop Traumatol Surg Res. 2012;98(6 Suppl):131-8.

62. Duzgun I, Baltaci G, Turgut E, Atay OA. Effects of slow and accelerated rehabilitation protocols on range of motion after arthroscopic rotator cuff repair. Acta Orthop Traumatol Turc. 2014;48(6):642-8.

63. Keener JD, Galatz LM, Stobbs-Cucchi G, Patton R, Yamaguchi K. Rehabilitation following arthroscopic rotator cuff repair: a prospective randomized trial of immobilization compared with early motion. J Bone Joint Surg Am. 2014;96(1):11-9.

64. Kim YS, Chung SW, Kim JY, Ok JH, Park I, Oh JH. Is early passive motion exercise necessary after arthroscopic rotator cuff repair? Am J Sports Med. 2012;40(4):815-21.

65. Kluczynski MA, Nayyar S, Marzo JM, Bisson LJ. Early versus delayed passive range of motion after rotator cuff repair: a systematic review and metaanalysis. Am J Sports Med. 2015;43(8):2057-63.

66. Kluczynski MA, Isenburg MM, Marzo JM, Bisson L. Does early versus delayed active range of motion affect rotator cuff healing after surgical repair? A systematic review and meta-analysis. Am J Sports Med. 2016;44(3):785-91.

67. Lee $B G$, Cho NS, Rhee YG. Effect of two rehabilitation protocols on range of motion and healing rates after arthroscopic rotator cuff repair: aggressive versus limited early passive exercises. Arthroscopy. 2012;28(1):34-42.

68. Mazzocca AD, Arciero RA, Shea KP, Apostolakos JM, Solovyova O, Gomlinski G, Wojcik KE, Tafuto V, Stock H, Cote MP. The Effect of early range of motion on quality of life, clinical outcome, and repair integrity after arthroscopic rotator cuff repair. Arthroscopy. 2017;33(6):1138-48.

69. Raschhofer R, Poulios N, Schimetta W, Kisling R, Mittermaier C. Early active rehabilitation after arthroscopic rotator cuff repair: a prospective randomized pilot study. Clin Rehabil. 2017;31(10):1332-9.

70. Cuff DJ, Pupello DR. Prospective randomized study of arthroscopic rotator cuff repair using an early versus delayed postoperative physical therapy protocol. J Shoulder Elbow Surg. 2012;21(11):1450-5.

71. Düzgün I, Baltacı G, Atay OA. Comparison of slow and accelerated rehabilitation protocol after arthroscopic rotator cuff repair: pain and functional activity. Acta Orthop Traumatol Turc. 2011;45(1):23-33.

72. Klintberg $\mathrm{H}$, Gunnarsson AC, Svantesson U, Styf J, Karlsson J. Early loading in physiotherapy treatment after full-thickness rotator cuff repair: a prospective randomized pilot-study with a two-year follow-up. Clin Rehabil. 2009:23(7):622-38.

73. Jenssen KK, Lundgreen K, Madsen JE, Kvakestad R, Pripp AH, Dimmen S. No functional difference between three and six weeks of immobilization after arthroscopic rotator cuff repair: a prospective randomized controlled noninferiority trial. Arthroscopy. 2018;34(10):2765-74.

74. Raman J, Walton D, MacDermid JC, Athwal GS. Predictors of outcomes after rotator cuff repair-a meta-analysis. J Hand Ther. 2017;30(3):276-92.

75. Peltz CD, Sarver JJ, Dourte LM, Würgler-Hauri CC, Williams GR, Soslowsky LJ. Exercise following a short immobilization period is detrimental to tendon properties and joint mechanics in a rat rotator cuff injury model. J Orthop Res. 2010;28(7):841-5.

76. Gimbel JA, Van Kleunen JP, Williams GR, Thomopoulos S, Soslowsky $\sqcup$. Long durations of immobilization in the rat result in enhanced mechanical properties of the healing supraspinatus tendon insertion site. J Biomech Eng. 2007;129(3):400-4. 
77. Koh KH, Lim TK, Shon MS, Park YE, Lee SW, Yoo JC. Effect of immobilization without passive exercise after rotator cuff repair: randomized clinical trial comparing four and eight weeks of immobilization. J Bone Joint Surg Am Vol 2014;96(6):e44

78. Sheps DM, Bouliane M, Styles-Tripp F, Beaupre LA, Saraswat MK, Luciak-Corea C, Silveira A, Glasgow R, Balyk R. Early mobilisation following mini-open rotator cuff repair: a randomised control trial. Bone Joint J. 2015;97-B(9):1257-63.

79. Sheps DM, Silveira A, Beaupre L, Styles-Tripp F, Balyk R, Lalani A, Glasgow R, Bergman J, Bouliane M, (SURGE) SaUERGoE. Early active motion versus sling immobilization after arthroscopic rotator cuff repair: a randomized controlled trial. Arthroscopy. 2019;35(3):749-60.e742.

80. Tirefort J, Schwitzguebel AJ, Collin P, Nowak A, Plomb-Holmes C, Ladermann A. Postoperative mobilization after superior rotator cuff repair: sling versus no sling: a randomized prospective study. J Bone Joint Surg Am Vol. 2019;101(6):494-503.

81. Hatakeyama Y, Itoi E, Pradhan RL, Urayama M, Sato K. Effect of arm elevation and rotation on the strain in the repaired rotator cuff tendon. A cadaveric study. Am J Sports Med. 2001;29(6):788-94.

82. Conti M, Garofalo R, Castagna A. Does a brace influence clinical outcomes after arthroscopic rotator cuff repair? Musculoskelet Surg. 2015;99(Suppl 1):31-5.

83. Hollman F, Wolterbeek N, Zijl JAC, van Egeraat SPM, Wessel RN. Abduction brace versus antirotation sling after arthroscopic cuff repair: the effects on pain and function. Arthroscopy. 2017;33(9):1618-26.

84. van der Meijden OA, Westgard P, Chandler Z, Gaskill TR, Kokmeyer D, Millett PJ. Rehabilitation after arthroscopic rotator cuff repair: current concepts review and evidence-based guidelines. Int J Sports Phys Ther. 2012;7(2):197218.

85. Muraki T, Aoki M, Uchiyama E, Miyasaka T, Murakami G, Miyamoto S. Strain on the repaired supraspinatus tendon during manual traction and translational glide mobilization on the glenohumeral joint: a cadaveric biomechanics study. Man Ther. 2007;12(3):231-9.

86. Johnson AJ, Godges JJ, Zimmerman GJ, Ounanian LL. The effect of anterior versus posterior glide joint mobilization on external rotation range of motion in patients with shoulder adhesive capsulitis. J Orthop Sports Phys Ther. 2007;37(3):88-99.

87. Koo SS, Burkhart SS. Rehabilitation following arthroscopic rotator cuff repair. Clin Sports Med. 2010;29(2):203-11. vii.

88. Peltz CD, Sarver JJ, Dourte LM, Wurgler-Hauri CC, Williams GR, Soslowsky LJ. Exercise following a short immobilization period is detrimental to tendon properties and joint mechanics in a rat rotator cuff injury model. J Orthop Res. 2010;28(7):841-5.

89. Abtahi AM, Granger EK, Tashjian RZ. Factors affecting healing after arthroscopic rotator cuff repair. World J Orthop. 2015:6(2):211-20.

90. Boileau P, Brassart N, Watkinson DJ, Carles M, Hatzidakis AM, Krishnan SG. Arthroscopic repair of full-thickness tears of the supraspinatus: does the tendon really heal? J Bone Joint Surg Am. 2005;87(6):1229-40.

91. Harryman DT, Mack LA, Wang KY, Jackins SE, Richardson ML, Matsen FA. Repairs of the rotator cuff. Correlation of functional results with integrity of the cuff. J Bone Joint Surg Am. 1991;73(7):982-9.

92. Yi A, Villacis D, Yalamanchili R, Hatch GF. A comparison of rehabilitation methods after arthroscopic rotator cuff repair: a systematic review. Sports Health. 2015;7(4):326-34.

93. Thomson $\mathrm{S}$, Jukes $\mathrm{C}$, Lewis J. Rehabilitation following surgical repair of the rotator cuff: a systematic review. Physiotherapy. 2016;102(1):20-8.

94. Marik TL, Roll SC. Effectiveness of occupational therapy interventions for musculoskeletal shoulder conditions: a systematic review. Am J Occup Ther. 2017;71(1):7101180020p7101180021-11.

95. Chan K, MacDermid JC, Hoppe DJ, Ayeni OR, Bhandari M, Foote CJ, Athwal GS. Delayed versus early motion after arthroscopic rotator cuff repair: a meta-analysis. J Shoulder Elbow Surg. 2014;23(11):1631-9.

96. Chang KV, Hung CY, Han DS, Chen WS, Wang TG, Chien KL. Early versus delayed passive range of motion exercise for arthroscopic rotator cuff repair: a meta-analysis of randomized controlled trials. Am J Sports Med. 2015:43(5):1265-73.

97. Littlewood C, Bateman M, Clark D, Selfe J, Watkinson D, Walton M, Funk L. Rehabilitation following rotator cuff repair: a systematic review. Shoulder Elbow. 2015;7(2):115-24.

98. Saltzman BM, Zuke WA, Go B, Mascarenhas R, Verma NN, Cole BJ, Romeo $A A$, Forsythe B. Does early motion lead to a higher failure rate or better outcomes after arthroscopic rotator cuff repair? A systematic review of overlapping meta-analyses. J Shoulder Elbow Surg. 2017;26(9):1681-91.
99. Page MJ, McKenzie JE, Bossuyt PM, Boutron I, Hoffmann TC, Mulrow CD, Shamseer L, Tetzlaff JM, Moher D. Updating guidance for reporting systematic reviews: development of the PRISMA 2020 statement. J Clin Epidemiol. 2021;134:103-12.

100. Schwitzguebel AJ, Kolo FC, Tirefort J, Kourhani A, Nowak A, Gremeaux V, Saffarini M, Ladermann A. Efficacy of platelet-rich plasma for the treatment of interstitial supraspinatus tears: a double-blinded, randomized controlled trial. Am J Sports Med. 2019;47(8):1885-92.

101. Cumpston M, Li T, Page MJ, Chandler J, Welch VA, Higgins JP, Thomas J. Updated guidance for trusted systematic reviews: a new edition of the Cochrane Handbook for Systematic Reviews of Interventions. Cochrane Database Syst Rev. 2019;10:ED000142

102. Landis JR, Koch GG. The measurement of observer agreement for categorical data. Biometrics. 1977;33(1):159-74

103. Guyatt GH, Oxman AD, Vist G, Kunz R, Brozek J, Alonso-Coello P, Montori V, Akl EA, Djulbegovic B, Falck-Ytter Y, et al. GRADE guidelines: 4. Rating the quality of evidence-study limitations (risk of bias). J Clin Epidemiol. 2011; 64(4):407-15.

104. Lastayo PC, Wright T, Jaffe R, Hartzel J. Continuous passive motion after repair of the rotator cuff. A prospective outcome study. J Bone Joint Surg Am. 1998;80(7):1002-11.

105. Raab MG, Rzeszutko D, O'Connor W, Greatting MD. Early results of continuous passive motion after rotator cuff repair: a prospective, randomized, blinded, controlled study. Am J Orthop (Belle Mead NJ). 1996; 25(3):214-20.

106. Michael JW, Konig DP, Imhoff AB, Martinek V, Braun S, Hubscher M, Koch C, Dreithaler B, Bernholt J, Preis $S$, et al. [Efficiency of a postoperative treatment after rotator cuff repair with a continuous passive motion device (CPM)]. Z Orthop Ihre Grenzgeb. 2005;143(4):438-45.

107. Garofalo R, Conti M, Notarnicola A, Maradei L, Giardella A, Castagna A Effects of one-month continuous passive motion after arthroscopic rotator cuff repair: results at 1-year follow-up of a prospective randomized study. Musculoskelet Surg. 2010;94(Suppl 1):79-83.

108. Buker N, Kitis A, Akkaya S, Akkaya N. Comparison of the results of supervised physiotherapy program and home-based exercise program in patients treated with arthroscopic-assisted mini-open rotator cuff repair. Eklem Hastalik Cerrahisi. 2011;22(3):134-9.

109. Deutsch A, Guelich DM, George, Govea C, Labis J. The effect of rehabilitation on cuff integrity and range of motion following arthroscopic rotator cuff repair: a prospective, randomized study of a standard vs. decelerated rehabilitation protocol (SS-23). In: vol. 16. Journal of Shoulder and Elbow Surgery: Elsevier Inc; 2007: e62.

110. De Roo PJ, Muermans S, Maroy M, Linden P, Van den Daelen L. Passive mobilization after arthroscopic rotator cuff repair is not detrimental in the early postoperative period. Acta Orthop Belg. 2015:81(3):485-92.

111. Simovitch R, Flurin PH, Wright T, Zuckerman JD, Roche CP. Quantifying success after total shoulder arthroplasty: the minimal clinically important difference. J Shoulder Elbow Surg. 2018;27(2):298-305.

112. Xu S, Chen JY, Lie HME, Hao Y, Lie DTT. Minimal clinically important difference of Oxford, Constant, and UCLA shoulder score for arthroscopic rotator cuff repair. J Orthop. 2020;19:21-7.

113. Schmidt L, Shokraneh F, Steinhausen K, Adams CE. Introducing RAPTOR: RevMan Parsing Tool for Reviewers. Syst Rev. 2019;8(1):151.

114. Tashjian RZ. AAOS clinical practice guideline: optimizing the management of rotator cuff problems. J Am Acad Orthop Surg. 2011;19(6):380-3.

115. Sarver JJ, Peltz CD, Dourte L, Reddy S, Williams GR, Soslowsky LJ. After rotator cuff repair, stiffness-but not the loss in range of motion-increased transiently for immobilized shoulders in a rat model. J Shoulder Elbow Surg. 2008;17(1 Suppl):108S-113S.

116. Carpenter JE, Thomopoulos S, Flanagan CL, DeBano CM, Soslowsky LJ. Rotator cuff defect healing: a biomechanical and histologic analysis in an animal model. J Shoulder Elbow Surg. 1998;7(6):599-605.

117. Mollison S, Shin JJ, Glogau A, Beavis RC. Postoperative rehabilitation after rotator cuff repair: a web-based survey of AANA and AOSSM members. Orthop J Sports Med. 2017;5(1):2325967116684775

118. Longo UG, Berton A, Khan WS, Maffulli N, Denaro V. Histopathology of rotator cuff tears. Sports Med Arthrosc Rev. 2011;19(3):227-36.

119. Gladstone JN, Bishop JY, Lo IK, Flatow EL. Fatty infiltration and atrophy of the rotator cuff do not improve after rotator cuff repair and correlate with poor functional outcome. Am J Sports Med. 2007;35(5): $719-28$. 
120. Liem D, Bartl C, Lichtenberg S, Magosch P, Habermeyer P. Clinical outcome and tendon integrity of arthroscopic versus mini-open supraspinatus tendon repair: a magnetic resonance imaging-controlled matched-pair analysis. Arthroscopy. 2007;23(5):514-21.

121. Bishop J, Klepps S, Lo IK, Bird J, Gladstone JN, Flatow EL. Cuff integrity after arthroscopic versus open rotator cuff repair: a prospective study. J Shoulder Elbow Surg. 2006;15(3):290-9.

122. Galatz LM, Ball CM, Teefey SA, Middleton WD, Yamaguchi K. The outcome and repair integrity of completely arthroscopically repaired large and massive rotator cuff tears. J Bone Joint Surg Am. 2004;86(2):219-24.

123. Chona DV, Lakomkin N, Lott A, Workman AD, Henry AC, Kuntz AF, Huffman GR, Glaser DL. The timing of retears after arthroscopic rotator cuff repair. J Shoulder Elbow Surg. 2017;26(11):2054-9.

124. Gumina S, Izzo R, Pintabona G, Candela V, Savastano R, Santilli V. Mobility recovery after arthroscopic rotator cuff repair. Eur J Phys Rehabil Med. 2017; 53(1):49-56.

125. Parsons BO, Gruson KI, Chen DD, Harrison AK, Gladstone J, Flatow EL. Does slower rehabilitation after arthroscopic rotator cuff repair lead to long-term stiffness? J Shoulder Elbow Surg. 2010;19(7):1034-9.

126. Nikolaidou O, Migkou S, Karampalis C. Rehabilitation after rotator cuff repair. Open Orthop J. 2017;11:154-62.

127. Thigpen CA, Shaffer MA, Kissenberth MJ. Knowing the speed limit: weighing the benefits and risks of rehabilitation progression after arthroscopic rotator cuff repair. Clin Sports Med. 2015;34(2):233-46.

128. Li S, Sun H, Luo X, Wang K, Wu G, Zhou J, Wang P, Sun X. The clinical effect of rehabilitation following arthroscopic rotator cuff repair: a meta-analysis of early versus delayed passive motion. Medicine. 2018;97(2):e9625.

\section{Publisher's Note}

Springer Nature remains neutral with regard to jurisdictional claims in published maps and institutional affiliations.

Ready to submit your research? Choose BMC and benefit from:

- fast, convenient online submission

- thorough peer review by experienced researchers in your field

- rapid publication on acceptance

- support for research data, including large and complex data types

- gold Open Access which fosters wider collaboration and increased citations

- maximum visibility for your research: over $100 \mathrm{M}$ website views per year

At $\mathrm{BMC}$, research is always in progress.

Learn more biomedcentral.com/submissions 